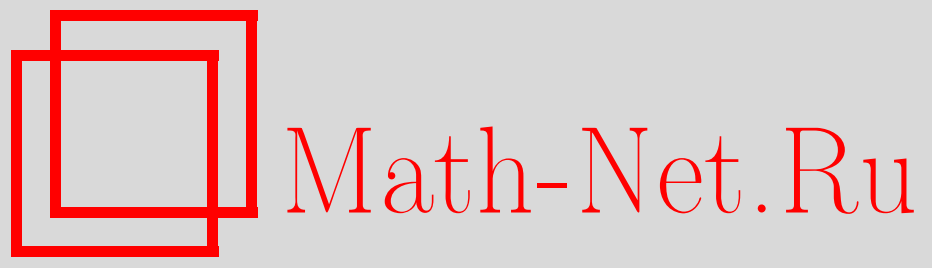

М. П. Савелов, Экстремальные характеристики критериев выбора гипотез с заданными попарными расстояниями по вариации, Теория вероятн. и ее примен., 2016, том 61, выпуск $3,439-463$

DOI: https://doi.org/10.4213/tvp5068

Использование Общероссийского математического портала Math-Net.Ru подразумевает, что вы прочитали и согласны с пользовательским соглашением http://www . mathnet.ru/rus/agreement

Параметры загрузки:

IP : 54.198 .64 .247

26 апреля 2023 г., $17: 18: 11$






\title{
ЭКСТРЕМАЛЬНЫЕ ХАРАКТЕРИСТИКИ КРИТЕРИЕВ ВЫБОРА ГИПОТЕЗ С ЗАДАННЫМИ ПОПАРНЫМИ РАССТОЯНИЯМИ ПО ВАРИАЦИИ
}

\begin{abstract}
Изучаются экстремальные значения характеристик нерандомизированных статистических критериев выбора одной из $n$ гипотез, когда известны только попарные расстояния по вариации между ними. Показано, что нахождение экстремальных значений функции общего вида от вероятностей ошибок сводится к решению конечномерной задачи линейного программирования. В случае $n=3$ и функции, равной сумме трех вероятностей ошибок, найдены точные формулы для ее экстремальных значений.

Ключевье слова и фразыл: различение многих гипотез, нерандомизированные критерии, расстояние по вариации, экстремальные наборы распределений.
\end{abstract}

1. Введение. Одной из важных статистических задач является задача о различении нескольких (скажем, $n$ ) простых гипотез. Если $n=2$, то ее решение (для широкого класса случаев) может быть получено с помощью леммы Неймана-Пирсона. Рассмотрим ситуацию, когда $n=3$. Для любой фиксированной тройки вероятностных мер $\vec{\mu}:=\left(\mu_{1}, \mu_{2}, \mu_{3}\right)$ можно найти оптимальный в том или ином смысле критерий и определить его «качество» (например, понимая под качеством вероятность ошибки в худшем случае) как функцию от тройки мер. В связи с этим естественным образом возникает вопрос: в каких пределах меняется качество критерия, если тройки вероятностных мер принадлежат некоторому множеству $D$. Формализуем вышесказанное.

Пусть $M$ - множество вероятностных мер на измеримом пространстве $(\Omega, \Sigma)$. Для любых трех вероятностных мер $\mu_{1}, \mu_{2}, \mu_{3}$ на $(\Omega, \Sigma)$ можно рассмотреть задачу различения трех гипотез $H_{i}$ : наблюдение $\xi$ имеет распределение $\mu_{i}(i=1,2,3)$. Каждому нерандомизированному критерию в этой задаче соответствуют измеримое разбиение $\vec{C}=$ $\left\{C_{1}, C_{2}, C_{3}\right\}$ пространства $\Omega\left(C_{i}: \bigsqcup_{i=1}^{3} C_{i}=\Omega\right.$, если наблюдение $\xi \in C_{i}$, то принимается гипотеза $\left.H_{i}, i=1,2,3\right)$ и вероятности $\mu_{j}\left(C_{k}\right)$ принятия

* Московский государственный университет им. М. В. Ломоносова, Москва, Россия; e-mail: savelovmp@gmail.com 
гипотезы $H_{k}$ в случае, когда верна гипотеза $H_{j}, j, k \in\{1,2,3\}$. Совокупность всех разбиений $\Omega$ на три непересекающихся измеримых подмножества обозначим $\mathscr{C}(\Omega)$. Далее, через $\vec{\mu}$ обозначим тройку $\left(\mu_{1}, \mu_{2}, \mu_{3}\right)$, через $\vec{\mu}(\vec{C})$ обозначим упорядоченный набор $\left(\mu_{1}\left(C_{1}\right), \mu_{1}\left(C_{2}\right), \ldots, \mu_{3}\left(C_{3}\right)\right)$. Будем считать, что качество критерия можно представить функцией $f(\vec{\mu}(\vec{C}))$ со значениями в $\mathbf{R}$; иногда будет использоваться также обозначение $f\left(\left\{\mu_{j}\left(C_{k}\right)\right\}_{j, k=1}^{3}\right)$. Фиксируем такие числа $r_{i j}, 1 \leqslant i<j \leqslant 3$, что $0 \leqslant r_{i j} \leqslant 1,1 \leqslant i<j \leqslant 3, r_{12}+r_{13} \geqslant r_{23}, r_{12}+r_{23} \geqslant r_{13}$ и $r_{13}+r_{23} \geqslant r_{12}$. Рассмотрим семейство всевозможных троек вероятностных мер на $\Sigma$, между которыми заданы попарные расстояния по вариации следующим образом: $\rho\left(\mu_{i}, \mu_{j}\right)=r_{i j}, 1 \leqslant i<j \leqslant 3$, где

$$
\rho\left(\mu_{i}, \mu_{j}\right):=\sup _{A \in \Sigma}\left|\mu_{i}(A)-\mu_{j}(A)\right| \text {. }
$$

Будем называть такие тройки мер $\vec{r}$-согласованными и обозначать это следующим образом: $\vec{\mu} \in D(\vec{r})$. Естественным образом возникает вопрос о получении удобных выражений для чисел $\bar{R}=$ $\sup _{\vec{\mu} \in D(\vec{r})} \sup _{\vec{C} \in \mathscr{C}(\Omega)} f(\vec{\mu}(\vec{C}))$ и $\underline{R}=\inf _{\vec{\mu} \in D(\vec{r})} \sup _{\vec{C} \in \mathscr{C}(\Omega)} f(\vec{\mu}(\vec{C}))$. Цель данной работы состоит в том, чтобы их найти. Отметим, что величины $\inf _{\vec{\mu} \in D(\vec{r})} \inf _{\vec{C} \in \mathscr{C}(\Omega)} f(\vec{\mu}(\vec{C}))$ и $\sup _{\vec{\mu} \in D(\vec{r})} \inf _{\vec{C} \in \mathscr{C}(\Omega)} f(\vec{\mu}(\vec{C}))$ сразу получаются из величин $\bar{R}$ и $\underline{R}$ за счет замены $f$ на $-f$.

Теперь мы можем сформулировать основные теоремы. Во-первых, мы рассмотрим конкретную функцию качества критерия, которая имеет вид $f(\vec{\mu}(\vec{C}))=\sum_{i=1}^{3} \mu_{i}\left(C_{i}\right)$. Выбор ее связан с тем, что, как показано в приведенной ниже лемме 4, оптимальным с точки зрения этой функции $f$ будет критерий, который тесно связан с оценкой максимального правдоподобия. Установлена следующая теорема.

Теорема 1. Пусть в б-алгебре измеримого пространства $(\Omega, \Sigma)$ содержится по крайней мере 18 непустьх попарно не пересекающихся множеств и пусть $f(\vec{\mu}(\vec{C}))=\sum_{i=1}^{3} \mu_{i}\left(C_{i}\right)$. Тогда для величин $\bar{R}^{+}=$ $\sup _{\vec{\mu} \in D(\vec{r})} \sup _{\vec{C} \in \mathscr{C}(\Omega)} \sum_{i=1}^{3} \mu_{i}\left(C_{i}\right) u \underline{R}^{+}=\inf _{\vec{\mu} \in D(\vec{r})} \sup _{\vec{C} \in \mathscr{C}(\Omega)} \sum_{i=1}^{3} \mu_{i}\left(C_{i}\right)$ Bblполняются следующие равенства:

$$
\begin{aligned}
& \bar{R}^{+}=1+\min \left(r_{13}+r_{23}, r_{12}+r_{23}, r_{12}+r_{13}\right), \\
& \underline{R}^{+}=\max \left(r_{12}+r_{13}+r_{23}, 1+\max \left(r_{12}, r_{13}, r_{23}\right)\right) .
\end{aligned}
$$

Более того, будет показано (см. теорему 5), что утверждение теоремы 1 не изменится и в случае, если экстремум берется по множеству рандомизированных критериев.

Также будет доказано, что в общем случае можно провести «понижение размерности», т.е. свести исходную задачу о нахождении чисел $\bar{R}$ и $\underline{R}$ к поиску экстремума функции на выпуклых замкнутых многогранниках $N_{18} \subset[0,1]^{54}$ и $N_{6} \subset[0,1]^{18}$, определенных формулами (3)-(5) и (7)-(9) соответственно. Справедлива следующая теорема. 
Теорема 2. Пусть в б-алгебре измеримого пространства $(\Omega, \Sigma)$ содержится по крайней мере 18 непустьх попарно не пересекающихся множеств. Тогда для любой функиии $f:[0,1]^{9} \rightarrow \mathbf{R}$ выполнены следующие равенства:

$$
\begin{aligned}
& \bar{R}=\sup _{\vec{x} \in N_{18}} f\left(\left\{\sum_{\sigma \in S_{3}} x_{\sigma}^{(j), k}\right\}_{j, k=1}^{3}\right), \\
& \underline{R}=\inf _{\vec{x} \in N_{6}} \max _{\vec{C} \in \mathscr{C}\left(S_{3}\right)} f\left(\left\{\sum_{\sigma \in C_{k}} x_{\sigma}^{(j)}\right\}_{j, k=1}^{3}\right),
\end{aligned}
$$

где $S_{3}$ - множество перестановок третьего порядка.

Другими словами, задача о нахождении $\bar{R}$ сводится к задаче о нахождении супремума по подмножеству $\mathbf{R}^{54}$, а задачу о нахождении $\underline{R}$ удается свести к задаче о нахождении минимакса в $\mathbf{R}^{18}$. Для непрерывных кусочно-линейных функций $f$ обе полученные формулы сводят задачу о нахождении $\bar{R}$ и $\underline{R}$ к задачам линейного программирования.

Ниже мы определим множества $Q_{6}, G_{6} \subset \mathbf{R}^{6}$ и докажем следующую теорему.

Теорема 3. Пусть в б-алгебре измеримого пространства $(\Omega, \Sigma)$ содержится по крайней мере 18 непустых попарно не пересекающихся множеств. Для любого $\vec{z}=\left(z_{1}, z_{2}, z_{3}, z_{4}, z_{5}, z_{6}\right) \in Q_{6} \cup G_{6}$ положим $f^{*}\left(z_{1}, z_{2}, z_{3}, z_{4}, z_{5}, z_{6}\right):=f\left(z_{1}, z_{2}, 1-z_{1}-z_{2}, z_{3}, z_{4}, 1-z_{3}-z_{4}, z_{5}, z_{6}, 1-z_{5}-z_{6}\right)$. Tогда

$$
\bar{R}=\sup _{\vec{z} \in Q_{6}} f^{*}\left(z_{1}, z_{2}, z_{3}, z_{4}, z_{5}, z_{6}\right), \quad \underline{R}=\inf _{\vec{z} \in G_{6}} f^{*}\left(z_{1}, z_{2}, z_{3}, z_{4}, z_{5}, z_{6}\right) .
$$

Отметим, что в теореме 2 множества, по которым берется экстремум (т.е. $N_{6}$ и $N_{18}$ ), устроены в некотором смысле проще, чем множества $G_{6}$ и $Q_{6}$ из теоремы 3 , однако последние, в отличие от первых, лежат в пространстве меньшей размерности.

Далее, будет доказана теорема 4, которая является простым обобщением теоремы 2 на случай произвольного конечного числа $(n)$ мер.

Также будет доказано, что при любом $n \geqslant 2$ существуют непрерывные функции $f$ качества критерия, для которых решение экстремальной задачи для $\underline{R}$ (в случае с $n$ мерами) в классе мер без атомов (более узком) качественно отличается от решения той же задачи в случае, когда наличие атомов у мер допускается (и $\Omega \neq \varnothing)$.

Наконец, заметим, что леммы 1 и 2, сформулированные ниже, доказывают, что если в $\sigma$-алгебре измеримого пространства $(\Omega, \Sigma)$ содержится по крайней мере 18 непустых попарно не пересекающихся множеств, то экстремум в задаче о нахождении чисел $\underline{R}$ и $\bar{R}$ достигается на множестве дискретных мер. 
2. Обсуждение. Одним из классических результатов в обсуждаемой тематике является лемма Фано (см. [6]), позволяющая оценить вероятности ошибок в терминах расстояния Кульбака-Лейблера. Усиление этого результата (также в терминах расстояния Кульбака-Лейблера) есть в статье [3]. Оценка вероятностей ошибок через более общие $f$ дивергентные расстояния приведена в статье [2], результаты которой позволяют усилить результаты статьи [3], а также получить следующие оценки вероятностей ошибок в терминах расстояний по вариации.

Пусть $\mathbb{E}=\left(\Omega, \mathbb{F},\left(P_{\theta}\right)_{\theta \in \Theta}\right)$ - статистическая модель, причем $\Theta=$ $\{1,2, \ldots, n\}$ и пространство $D$ решений совпадает с множеством $\Theta$, функция потерь $L(\theta, d)=\mathbb{I}_{\theta \neq d}$, функция $\phi=\left(\phi_{1}, \ldots, \phi_{n}\right)$ задает рандомизированный критерий $\left(\phi_{i}: \Omega \rightarrow[0,1]\right.$ измеримы для каждого $1 \leqslant$ $i \leqslant n$ и $\left.\sum_{i=1}^{n} \phi_{i}=1\right)$ следующим образом: $\phi_{i}(\omega)$ - это вероятность принять решение под номером $i$, если происходит $\omega$. Множество таких критериев обозначим $\Phi$. Далее, $a_{M}:=\sup _{\phi \in \Phi} \min _{1 \leqslant i \leqslant n} \mathbf{E}_{P_{i}} \phi_{i}$ (число $1-a_{M}$ равно вероятности ошибки «в худшем случае»). Положим $\bar{a}:=\sup _{\phi \in \Phi}(1 / n) \sum_{i=1}^{n} \mathbf{E}_{P_{i}} \phi_{i}$. С помощью теоремы 1 из статьи [2] легко получить следующую оценку:

$$
a_{M} \leqslant \bar{a} \leqslant \frac{1}{n}\left(1+\min _{j} \sum_{i=1}^{n} \rho\left(P_{i}, P_{j}\right)\right),
$$

которая совпадает с величиной $\bar{R}^{+}$в случае $n=3$. Мы докажем, что в условиях, когда $n=3$ и известны только расстояния по вариации между мерами, эта оценка является неулучшаемой, причем доказательство позволит одновременно получить нижнюю оценку для $\bar{a}$.

Заметим, что в случае, когда $n=2$ и критерии рандомизированные, величина $\bar{a}$ выражается через расстояние по вариации (см. $[1$, с. 461,462$])$ следующим образом: $\bar{a}=\left(1+\rho\left(P_{1}, P_{2}\right)\right) / 2$.

Ряд результатов, связывающих вероятность ошибки и характеристики соответствующих распределений (например, энтропию), представлен в работах [9], [10], [11]. Схожие вопросы рассмотрены также в статье [4], основная теорема которой состоит в следующем. Пусть $\mu_{1}, \ldots, \mu_{n}$ - безатомные вероятностные меры на измеримом пространстве $(S, \mathscr{B}), \Pi_{S}$ - множество измеримых разбиений $S$,

$$
\begin{aligned}
M\left(\mu_{1}, \ldots, \mu_{n}\right) & :=\sup \left\{\sum_{i=1}^{n} \mu_{i}\left(A_{i}\right):\left\{A_{i}\right\}_{i=1}^{n} \in \Pi_{S}\right\}, \\
v\left(\mu_{1}, \ldots, \mu_{n}\right) & :=\sup \left\{\min _{1 \leqslant i \leqslant n} \mu_{i}\left(A_{i}\right):\left\{A_{i}\right\}_{i=1}^{n} \in \Pi_{S}\right\},
\end{aligned}
$$

тогда $(n-M+1)^{-1} \leqslant v \leqslant M n^{-1}$.

Задача, решаемая в настоящей работе, отличается от вышеперечисленных тем, что, ограничившись только множеством $\Phi^{*}$ нерандомизи- 
рованных критериев, мы найдем величины $\underline{R}$ и $\bar{R}$, которые дают неулуч-

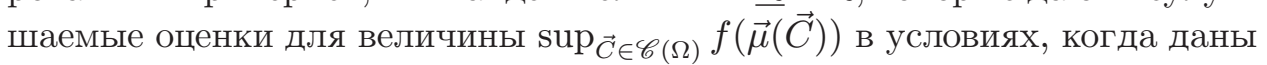
только расстояния по вариации $r_{i j}, 1 \leqslant i<j \leqslant 3$. Тогда неулучшаемые оценки для величины $a_{M}^{*}:=\sup _{\phi \in \Phi^{*}} \min _{1 \leqslant i \leqslant 3} \mathbf{E}_{P_{i}} \phi_{i}$ получаются как частный случай теоремы 2. В самом деле,

$$
a_{M}^{*}=\sup _{\phi \in \Phi^{*}} \min _{1 \leqslant i \leqslant 3} \mathbf{E}_{P_{i}} \phi_{i}=\sup _{\vec{C} \in \mathscr{C}(\Omega)} \min _{1 \leqslant i \leqslant 3}\left(\mu_{i}\left(C_{i}\right)\right) .
$$

Поэтому неулучшаемые оценки величины $a_{M}^{*}$ через тройку попарных расстояний $\rho\left(\mu_{i}, \mu_{j}\right)$ являются частным случаем выражений $\underline{R}$ и $\bar{R}$ при $f(\vec{\mu}(\vec{C}))=\min _{i} \mu_{i}\left(C_{i}\right)$. Далее, рассмотрим $\bar{a}^{*}=(1 / 3) \sup _{\phi \in \Phi^{*}} \sum_{i=1}^{3} \mathbf{E}_{P_{i}} \phi_{i}$. Очевидно, что $3 \bar{a}^{*}=\sup _{\vec{C} \in \mathscr{C}(\Omega)} \sum_{i}\left(\mu_{i}\left(C_{i}\right)\right)$, поэтому число $\bar{R}^{+}$будет неулучшаемой верхней оценкой величины $3 \bar{a}^{*}$ в случае, когда $f(\vec{\mu}(\vec{C}))=$ $\sum_{i} \mu_{i}\left(C_{i}\right)$, при этом (см. теорему 1) $\bar{R}^{+}=1+\min \left(r_{13}+r_{23}, r_{12}+r_{23}, r_{12}+\right.$ $\left.r_{13}\right)$. Более того, $\bar{a}=\bar{a}^{*}$ (см. доказательство теоремы 5$)$, и (в силу теоремы 5 ) величина $\bar{R}^{+}$является также неулучшаемой верхней оценкой величины $\bar{a}$. Аналогично величина $\underline{R}^{+}$является неулучшаемой нижней оценкой величин $3 \bar{a}$ и $3 \bar{a}^{*}$. Другими словами, из результатов статьи [2], примененной к случаю, когда заданы 3 меры, следуют верхние оценки величин $\bar{a}$ и $a_{M}$ в терминах расстояний по вариации между мерами, а из результатов настоящей статьи следуют неулучшаемые двусторонние оценки величин $\bar{a}, \bar{a}^{*}$ и $a_{M}^{*}$.

Отметим, наконец, что рассматриваемая тематика отчасти связана с проблемой деления торта (cake-cutting problem), см., например, [4]. В самом деле, задача о делении торта $(\Omega)$ на куски (измеримые множества $C_{i}$ ) между несколькими людьми (каждому человеку соответствует вероятностная мера $\left.\mu_{i}, 1 \leqslant i \leqslant n\right)$ может быть формализована, в частности, одним из следующих способов ([5, с. 62]). Можно назвать деление «честным», если, например, выполняется одно из следующих условий:

1) $\mu_{i}\left(C_{i}\right) \geqslant 1 / n$ для каждого $i, 1 \leqslant i \leqslant n$ (обычное честное деление),

2) $\mu_{i}\left(C_{i}\right) \geqslant \mu_{i}\left(C_{j}\right)$ для всех пар $1 \leqslant i, j \leqslant n$ («отсутствие зависти»).

Кроме того, естественно считать, что деление тем лучше, чем больше величина $\sum_{i=1}^{n} \mu_{i}\left(C_{i}\right)$ («суммарная удовлетворенность»). В рамках этих трех подходов естественно полагать, что деление тем лучше, чем больше значения следующих функций:

1) $h_{1}(\vec{\mu}(\vec{C}))=\min _{i} \mu_{i}\left(C_{i}\right)$,

2) $h_{2}(\vec{\mu}(\vec{C}))=\min _{i \neq j}\left(\mu_{i}\left(C_{i}\right)-\mu_{i}\left(C_{j}\right)\right)$,

3) $h_{3}(\vec{\mu}(\vec{C}))=\sum_{i} \mu_{i}\left(C_{i}\right)$.

Далее, естественным образом возникает задача о нахождении числовых характеристик оптимальных разбиений, т.е. задача о нахождении $\inf _{\vec{\mu} \in A} \sup _{\vec{C} \in \mathscr{C}(\Omega)} h_{l}(\vec{\mu}(\vec{C}))$ и $\sup _{\vec{\mu} \in A} \sup _{\vec{C} \in \mathscr{C}(\Omega)} h_{l}(\vec{\mu}(\vec{C})), 1 \leqslant l \leqslant 3$ (отметим, что функции $h_{l}$ непрерывны и кусочно-линейны), где $A-$ некое 
множество наборов из $n$ мер на $\Omega$. В случае, когда $A=D(\vec{r})$ (т.е. совпадает с множеством $\vec{r}$-согласованных мер) и в $\sigma$-алгебре измеримого пространства $(\Omega, \Sigma)$ содержится по крайней мере $n \cdot n !$ непустых попарно не пересекающихся множеств, решение данной задачи может быть получено с помощью теоремы 4.

Автор благодарит своего научного руководителя А. М. Зубкова за постановку задачи и постоянное внимание, а также А. А. Гущина за полезные советы и указания, позволившие усилить некоторые результаты.

3. Доказательство теоремы 2. Пусть $\vec{\mu}=\left(\mu_{1}, \mu_{2}, \mu_{3}\right)$ - тройка мер на измеримом пространстве $(\Omega, \Sigma)$. Будем называть множество $E$ отрицательным относительно некоторой знакопеременной меры $\Phi$, если $\Phi(E \bigcap F) \leqslant 0$ для любого $F \in \Sigma$; аналогично будем называть множество $E$ положительным относительно $\Phi$, если $\Phi(E \bigcap F) \geqslant 0$ для любого $F \in \Sigma$. В силу теоремы Хана о разложении (для знакопеременной меры $\left.\mu_{1}-\mu_{2}\right)$ найдется такое измеримое множество $R_{1}$, что оно положительно относительно $\mu_{1}-\mu_{2}$, а его дополнение $\Omega \backslash R_{1}$ отрицательно. Другими словами, для любого $F \in \Sigma$ выполнены следующие неравенства: $\mu_{1}\left(R_{1} \bigcap F\right) \geqslant \mu_{2}\left(R_{1} \bigcap F\right)$ и $\mu_{2}\left(\left(\Omega \backslash R_{1}\right) \bigcap F\right) \geqslant \mu_{1}\left(\left(\Omega \backslash R_{1}\right) \bigcap F\right)$. Аналогично (в силу теоремы Хана о разложении) найдутся такие множества $R_{2}$ и $R_{3}$, что $\mu_{2}\left(R_{2} \bigcap F\right) \geqslant \mu_{3}\left(R_{2} \bigcap F\right), \mu_{3}\left(\left(\Omega \backslash R_{2}\right) \bigcap F\right) \geqslant \mu_{2}\left(\left(\Omega \backslash R_{2}\right) \bigcap F\right)$, $\mu_{1}\left(R_{3} \cap F\right) \geqslant \mu_{3}\left(R_{3} \bigcap F\right)$ и, наконец, $\mu_{3}\left(\left(\Omega \backslash R_{3}\right) \bigcap F\right) \geqslant \mu_{1}\left(\left(\Omega \backslash R_{3}\right) \bigcap F\right)$. Положим по определению

$$
\begin{gathered}
B_{123}:=R_{1} R_{2} R_{3}, \quad B_{132}:=R_{1} \bar{R}_{2} R_{3}, \\
B_{312}:=R_{1} \bar{R}_{2} \bar{R}_{3}, \quad B_{213}:=\bar{R}_{1} R_{2} R_{3}, \\
B_{231}:=\bar{R}_{1} R_{2} \bar{R}_{3}, \quad B_{321}:=\bar{R}_{1} \bar{R}_{2} \bar{R}_{3}, \\
\quad\left(R_{1} R_{2} \bar{R}_{3}=\varnothing, \bar{R}_{1} \bar{R}_{2} R_{3}=\varnothing\right),
\end{gathered}
$$

где последние равенства можно считать выполненными без ограничения общности (в самом деле, если множество $R_{1} R_{2} \bar{R}_{3}$ или $\bar{R}_{1} \bar{R}_{2} R_{3}$ не является пустым, то его можно «присоединить» к $\left.R_{1}\right)$. Пусть $S_{3}=$ $\{123,132,213,231,312,321\}$ - множество перестановок (индексов множеств $\left.B_{i j k}\right)$. Несложно видеть, что набор множеств $B_{\sigma}, \sigma \in S_{3}$, является разбиением пространства $\Omega$, и если $\sigma=\left(\sigma_{1}, \sigma_{2}, \sigma_{3}\right) \in S_{3}$, то $\mu_{\sigma_{1}}\left(F \cap B_{\sigma}\right) \geqslant \mu_{\sigma_{2}}\left(F \cap B_{\sigma}\right) \geqslant \mu_{\sigma_{3}}\left(F \cap B_{\sigma}\right)$ при всех $F \in \Sigma$. Таким образом, для каждой тройки мер определены множества $B_{\sigma}(\vec{\mu}, \Omega)$, задающие разбиение пространства $\Omega$, на котором эти меры заданы. Далее, нам потребуется новое обозначение. Пусть $\sigma=\left(\sigma_{1} \sigma_{2} \sigma_{3}\right) \in S_{3}$, тогда $\sigma_{i}$ $(1 \leqslant i \leqslant 3)$ - это числа от 1 до 3 . Рассмотрим подстановку, которая переводит единицу в $\sigma_{1}$, двойку в $\sigma_{2}$, а тройку в $\sigma_{3}$. Тогда обратная ей переводит число $i$ в число, которое мы будем обозначать $\sigma_{i}^{-1}$. 
Заметим, что расстояния по вариации между мерами $\mu_{1}, \mu_{2}, \mu_{3}$ определяются значениями $\mu_{i}\left(B_{\sigma}\right)$, так как

$$
\begin{aligned}
2 r_{i j} & =\sum_{\sigma \in S_{3}}\left|\mu_{i}\left(B_{\sigma}\right)-\mu_{j}\left(B_{\sigma}\right)\right| \\
& =\sum_{\sigma \in S_{3}}\left(\mu_{i}\left(B_{\sigma}\right)-\mu_{j}\left(B_{\sigma}\right)\right)(-1)^{I\left\{\sigma_{i}^{-1}>\sigma_{j}^{-1}\right\}}, \quad 1 \leqslant i<j \leqslant 3 .
\end{aligned}
$$

Каждое разбиение $\vec{C}=\left\{C_{1}, C_{2}, C_{3}\right\}$ пространства $\Omega$, вообще говоря, разбивает каждое множество $B_{\sigma}$ на три подмножества $B_{\sigma}^{j}=B_{\sigma} \bigcap C_{j}$, $1 \leqslant j \leqslant 3$, и набор аргументов $\vec{\mu}(\vec{C})$ функции $f$ полностью определяется 54 значениями $\mu_{i}\left(B_{\sigma}^{j}\right): \mu_{i}\left(C_{j}\right)=\sum_{\sigma \in S_{3}} \mu_{i}\left(B_{\sigma}^{j}\right)$. Поэтому задача поиска экстремальных значений $f(\vec{\mu}(\vec{C}))$ сводится к задаче поиска экстремума на подмножестве $\mathbf{R}^{54}$, определяемом условиями на то, что меры $\mu_{1}, \mu_{2}, \mu_{3}$ - вероятностные с заданными расстояниями по вариации (и неравенствами, входящими в определения множеств $\left.B_{\sigma}\right)$.

Теперь перейдем к вопросу о нахождении $\bar{R}$. Рассмотрим пространство $\Omega_{18}=\left\{\omega_{\sigma}^{m}, \sigma \in S_{3}, 1 \leqslant m \leqslant 3\right\}$, состоящее из 18 элементов, и $\sigma$ алгебру $2^{\Omega_{18}}$ на нем. Через $Z_{18}$ обозначим семейство $\vec{r}$-согласованных троек мер $\mu_{1}, \mu_{2}, \mu_{3}$ на $\Omega_{18}$, т.е. $Z_{18}=\left\{\left\{\mu_{i}\right\}_{i=1}^{3}: \rho\left(\mu_{s}, \mu_{l}\right)=r_{s l}, 1 \leqslant s<\right.$ $l \leqslant 3\}$. Рассмотрим (по аналогии с введением множеств $B_{i j k}$ ) те тройки мер из $Z_{18}$, для которых при любых $1 \leqslant m \leqslant 3$ и $\sigma \in S_{3}$ выполнены следующие условия:

$$
\mu_{\sigma_{1}}\left(\omega_{\sigma}^{m}\right) \geqslant \mu_{\sigma_{2}}\left(\omega_{\sigma}^{m}\right) \geqslant \mu_{\sigma_{3}}\left(\omega_{\sigma}^{m}\right)
$$

Множество таких троек мер обозначим $\Lambda_{18}$. Далее, рассмотрим разбиение $\vec{C}^{0}:=\left\{C_{1}^{0}, C_{2}^{0}, C_{3}^{0}\right\}$, где $C_{m}^{0}=\bigsqcup_{\sigma \in S_{3}} \omega_{\sigma}^{m}$. Напомним, что по определению $\bar{R}=\sup _{\vec{\mu} \in D(\vec{r})} \sup _{\vec{C} \in \mathscr{C}(\Omega)} f(\vec{\mu}(\vec{C}))$. Верна следующая лемма.

Лемма 1. Пусть в б-алгебре измеримого пространства $(\Omega, \Sigma)$ содержится по крайней мере 18 непустых попарно не пересекающихся множеств $K_{\sigma}^{i}, \sigma \in S_{3}, 1 \leqslant i \leqslant 3$. Тогда для любой функиии $f:[0,1]^{9} \rightarrow \mathbf{R}$ виполняется следующее равенство:

$$
\bar{R}=\sup _{\vec{\mu} \in \Lambda_{18}} f\left(\vec{\mu}\left(\vec{C}^{0}\right)\right) .
$$

Д о к а з а т е л с с в о. Докажем сначала, что

$$
\bar{R} \geqslant \sup _{\vec{\mu} \in \Lambda_{18}} f\left(\vec{\mu}\left(\vec{C}^{0}\right)\right)
$$

Так как $K_{\sigma}^{i} \neq \varnothing, 1 \leqslant i \leqslant 3, \sigma \in S_{3}$, то существуют такие элементы $v_{i, \sigma}$, что $v_{i, \sigma} \in K_{\sigma}^{i}, 1 \leqslant i \leqslant 3, \sigma \in S_{3}$. Для любой $\vec{r}$ согласованной тройки $\vec{\mu}$ мер на $\Omega_{18}$ построим соответствующую тройку 
$\vec{\mu}^{*}$ мер на $\Omega$ следующим образом: $\mu_{i}^{*}(A)=\sum_{(m, \sigma): v_{m, \sigma} \in A} \mu_{i}\left(\omega_{\sigma}^{m}\right)$ при любом $A \in \Sigma$. Заметим, что $\sup _{\vec{C} \in \mathscr{C}\left(\Omega_{18}\right)} f(\vec{\mu}(\vec{C}))=\sup _{\vec{C} \in \mathscr{C}(\Omega)} f\left(\vec{\mu}^{*}(\vec{C})\right)$ и $\vec{\mu}^{*} \in D(\vec{r})$. Другими словами, для любой $\vec{r}$-согласованной тройки $\vec{\mu}$ мер на $\Omega_{18}$ найдется такая $\vec{r}$-согласованная тройка $\vec{\mu}^{*}$ мер на $\Omega$, что $\sup _{\vec{C} \in \mathscr{C}\left(\Omega_{18}\right)} f(\vec{\mu}(\vec{C}))=\sup _{\vec{C} \in \mathscr{C}(\Omega)} f\left(\vec{\mu}^{*}(\vec{C})\right)$. Значит, $\sup _{\vec{\mu} \in D(\vec{r})} \sup _{\vec{C} \in \mathscr{C}(\Omega)} f(\vec{\mu}(\vec{C})) \geqslant \sup _{\vec{\mu} \in Z_{18}} \sup _{\vec{C} \in \mathscr{C}\left(\Omega_{18}\right)} f(\vec{\mu}(\vec{C}))$. Далее, несложно видеть, что

$$
\sup _{\vec{\mu} \in Z_{18}} \sup _{\vec{C} \in \mathscr{C}\left(\Omega_{18}\right)} f(\vec{\mu}(\vec{C})) \geqslant \sup _{\vec{\mu} \in Z_{18}} f\left(\vec{\mu}\left(\vec{C}^{0}\right)\right) \geqslant \sup _{\vec{\mu} \in \Lambda_{18}} f\left(\vec{\mu}\left(\vec{C}^{0}\right)\right)
$$

в силу того, что $\Lambda_{18} \subset Z_{18}$. Значит,

$$
\sup _{\vec{\mu} \in D(\vec{r})} \sup _{\vec{C} \in \mathscr{C}(\Omega)} f(\vec{\mu}(\vec{C})) \geqslant \sup _{\vec{\mu} \in Z_{18}} \sup _{\vec{C} \in \mathscr{C}\left(\Omega_{18}\right)} f(\vec{\mu}(\vec{C})) \geqslant \sup _{\vec{\mu} \in \Lambda_{18}} f\left(\vec{\mu}\left(\vec{C}^{0}\right)\right),
$$

причем в левой части последнего неравенства стоит не что иное, как величина $\bar{R}$. Таким образом, $\bar{R} \geqslant \sup _{\vec{\mu} \in \Lambda_{18}} f\left(\vec{\mu}\left(\vec{C}^{0}\right)\right)$, и нам осталось доказать, что $\bar{R} \leqslant \sup _{\vec{\mu} \in \Lambda_{18}} f\left(\vec{\mu}\left(\vec{C}^{0}\right)\right)$. В самом деле, рассмотрим любую тройку $\vec{\mu} \in D(\vec{r})$ мер на $\Omega$ и любое разбиение $\vec{C}$ пространства $\Omega$. Построим тройку $\vec{\mu}^{*}$ мер на пространстве $\Omega_{18}$ следующим образом: $\mu_{i}^{*}\left(\omega_{\sigma}^{m}\right):=\mu_{i}\left(B_{\sigma} \bigcap C_{m}\right)$, где множества $B_{\sigma}=B_{\sigma}(\vec{\mu}, \Omega)$ строятся на основании разложения Хана так, как это сделано выше. Напомним, что по определению $C_{m}^{0}:=\bigsqcup_{\sigma} \omega_{\sigma}^{m}$, значит, $\mu_{i}\left(C_{m}\right)=\sum_{\sigma \in S_{3}} \mu_{i}\left(B_{\sigma} \cap C_{m}\right)=$ $\sum_{\sigma \in S_{3}} \mu_{i}^{*}\left(\omega_{\sigma}^{m}\right)=\mu_{i}^{*}\left(C_{m}^{0}\right)$, поэтому $\vec{\mu}(\vec{C})=\vec{\mu}^{*}\left(\vec{C}^{0}\right)$, откуда $f(\vec{\mu}(\vec{C}))=$ $f\left(\vec{\mu}^{*}\left(\vec{C}^{0}\right)\right)$. Кроме того, из $\vec{r}$-согласованности тройки мер $\vec{\mu}$ следует (в силу непосредственной проверки) $\vec{r}$-согласованность тройки $\vec{\mu}^{*}$. Убедимся, что $\vec{\mu}^{*} \in \Lambda_{18}$, проверив, что для этой тройки мер выполняется соотношение (1). В силу определения множеств $B_{\sigma}$ выполняются следующие неравенства:

$$
\begin{gathered}
\mu_{\sigma_{1}}\left(B_{\sigma} \cap C_{m}\right) \geqslant \mu_{\sigma_{2}}\left(B_{\sigma} \cap C_{m}\right) \geqslant \mu_{\sigma_{3}}\left(B_{\sigma} \cap C_{m}\right), \\
1 \leqslant m \leqslant 3, \quad \sigma=\left(\sigma_{1}, \sigma_{2}, \sigma_{3}\right) \in S_{3},
\end{gathered}
$$

значит $\mu_{\sigma_{1}}^{*}\left(\omega_{\sigma}^{m}\right) \geqslant \mu_{\sigma_{2}}^{*}\left(\omega_{\sigma}^{m}\right) \geqslant \mu_{\sigma_{3}}^{*}\left(\omega_{\sigma}^{m}\right)$ при всех $\sigma=\left(\sigma_{1}, \sigma_{2}, \sigma_{3}\right) \in S_{3}, 1 \leqslant$ $m \leqslant 3$. Итак, мы получили, что для каждой $\vec{r}$-согласованной тройки $\vec{\mu}$ мер на $\Omega$ и любого разбиения $\vec{C}$ пространства $\Omega$ найдется такая тройка $\vec{\mu}^{*}$ мер на $\Omega_{18}$, что $\vec{\mu}^{*} \in \Lambda_{18}, f(\vec{\mu}(\vec{C}))=f\left(\vec{\mu}^{*}\left(\vec{C}^{0}\right)\right)$. Отсюда сразу следует, что

$$
\sup _{\vec{\mu} \in D(\vec{r})} \sup _{\vec{C} \in \mathscr{C}(\Omega)} f(\vec{\mu}(\vec{C})) \leqslant \sup _{\vec{\mu} \in \Lambda_{18}} f\left(\vec{\mu}\left(\vec{C}^{0}\right)\right)
$$

причем в левой части последнего неравенства стоит, очевидно, величина $\bar{R}$. Таким образом, $\bar{R} \leqslant \sup _{\vec{\mu} \in \Lambda_{18}} f\left(\vec{\mu}\left(\vec{C}^{0}\right)\right)$. Лемма 1 полностью доказана. Заметим, что лемма 1 существенно упрощает задачу о нахождении числа $\bar{R}$ : можно считать, что $\Omega=\Omega_{18}, \Sigma=2^{\Omega_{18}}$ и тройка мер $\left(\mu_{1}, \mu_{2}, \mu_{3}\right)$ 
принадлежит множеству $\Lambda_{18}$. K тому же, лемма 1 позволяет избавиться от инфимума по разбиениям.

Теперь докажем первую формулу (для величины $\bar{R}$ ) из теоремы 2 , т.е. сведем задачу о нахождении $\bar{R}$ к поиску условного экстремума в $\mathbf{R}^{54}$ (предполагая, что условие теоремы 2 выполнено). Для этого фиксируем произвольную тройку мер $\left\{\mu_{l}\right\}_{l=1}^{3}$ из $\Lambda_{18}$ и любое разбиение $\vec{C}$ пространства $\Omega_{18}$. По тройке мер построим множества $B_{\sigma}\left(\vec{\mu}, \Omega_{18}\right)$. Введем обозначение $D_{\sigma}^{m}=C_{m} \bigcap B_{\sigma}, x_{\sigma}^{(l), m}=\mu_{l}\left(D_{\sigma}^{m}\right)$. Тогда на множествах $D_{i j k}^{m}$ выполнены неравенства $\mu_{i}\left(D_{i j k}^{m}\right) \geqslant \mu_{j}\left(D_{i j k}^{m}\right) \geqslant \mu_{k}\left(D_{i j k}^{m}\right)$. (Кроме того, несложно видеть, что на множествах $D_{i j k}^{m}, 1 \leqslant m \leqslant 3$, принимается $m$-я гипотеза.) Отсюда получаем соотношения между числами $x_{i j k}^{(l), m}$ (для всех $\left.1 \leqslant m \leqslant 3, \sigma \in S_{3}\right)$ :

$$
x_{\sigma}^{\left(\sigma_{1}\right), m} \geqslant x_{\sigma}^{\left(\sigma_{2}\right), m} \geqslant x_{\sigma}^{\left(\sigma_{3}\right), m} .
$$

Далее, для любых $\sigma \in S_{3}, F \in 2^{\Omega_{18}}$ выполнены неравенства $\mu_{\sigma_{1}}\left(B_{\sigma} \bigcap F\right) \geqslant$ $\mu_{\sigma_{2}}\left(B_{\sigma} \bigcap F\right) \geqslant \mu_{\sigma_{3}}\left(B_{\sigma} \bigcap F\right)$ и в силу $\vec{r}$-согласованности

$$
\begin{aligned}
2 r_{i j} & =\sum_{\sigma \in S_{3}}\left|\mu_{i}\left(B_{\sigma}\right)-\mu_{j}\left(B_{\sigma}\right)\right| \\
& =\sum_{\sigma \in S_{3}}\left(\mu_{i}\left(B_{\sigma}\right)-\mu_{j}\left(B_{\sigma}\right)\right) \cdot(-1)^{I\left\{\sigma_{i}^{-1}>\sigma_{j}^{-1}\right\}}, \quad 1 \leqslant i<j \leqslant 3 .
\end{aligned}
$$

Обозначим $x_{\sigma}^{(l)}:=\sum_{m=1}^{3} x_{\sigma}^{(l), m}$. Тогда $x_{\sigma}^{(l)}=\sum_{m=1}^{3} \mu_{l}\left(C_{m} \bigcap B_{\sigma}\right)=\mu_{l}\left(B_{\sigma}\right)$. Таким образом, расписывая условие $\vec{r}$-согласованности, получаем

$$
2 r_{i j}=\sum_{\sigma \in S_{3}}\left(x_{\sigma}^{(i)}-x_{\sigma}^{(j)}\right)(-1)^{I\left\{\sigma_{i}^{-1}>\sigma_{j}^{-1}\right\}}, \quad 1 \leqslant i<j \leqslant 3 .
$$

Кроме того, так как $\mu_{i}$ являются вероятностными мерами, то

$$
\sum_{\sigma \in S_{3}} x_{\sigma}^{(l)}=1, \quad 0 \leqslant x_{\sigma}^{(l)} \leqslant 1, \quad 1 \leqslant l \leqslant 3, \quad \sigma \in S_{3} .
$$

Значит,

$$
\begin{gathered}
2 r_{i j}=\sum_{\sigma \in S_{3}}\left(\sum_{m} x_{\sigma}^{(i), m}-\sum_{m} x_{\sigma}^{(j), m}\right)(-1)^{I\left\{\sigma_{i}^{-1}>\sigma_{j}^{-1}\right\}}, \quad 1 \leqslant i<j \leqslant 3, \\
\sum_{\sigma \in S_{3}} \sum_{m} x_{\sigma}^{(l), m}=1, \quad 0 \leqslant x_{\sigma}^{(l), m} \leqslant 1, \quad 1 \leqslant l \leqslant 3, \quad 1 \leqslant m \leqslant 3, \quad \sigma \in S_{3} .
\end{gathered}
$$

Пусть $\mathbb{T}$ - множество троек произвольных отображений $T_{i}$, $T_{i}: \Omega_{18} \rightarrow[0,1], 1 \leqslant i \leqslant 3$. Пусть $\phi_{18}: \mathbb{T} \rightarrow[0,1]^{54}$ и $\phi_{18}\left(T_{1}, T_{2}, T_{3}\right)=\vec{x}$, где 
$\vec{x}=\left(x_{123}^{(1), 1}, x_{123}^{(1), 2}, x_{123}^{(1), 3}, x_{123}^{(2), 1}, \ldots, x_{321}^{(3), 1}, x_{321}^{(3), 2}, x_{321}^{(3), 3}\right)$ и $x_{i j k}^{(l), m}=T_{l}\left(\omega_{i j k}^{m}\right)$. Несложно видеть, что такое отображение является биективным (как отображение из $\mathbb{T}$ в $\left.[0,1]^{54}\right)$. Далее, обозначим через $N_{18} \subset[0,1]^{54}$ множество векторов $\vec{x}$, удовлетворяющих условиям (3)-(5). Заметим, что отображение $\phi_{18}$ устанавливает биекцию между множеством $\Lambda_{18}$ и множеством $N_{18}$, так как условия принадлежности тройки мер множеству $\Lambda_{18}$ равносильны условиям (3)-(5) принадлежности вектора $\vec{x}$ множеству $N_{18}$. Далее, множество $N_{18} \subset[0,1]^{54}$ представимо в виде конечного пересечения (замкнутых) полупространств и гиперплоскостей, пересечение замкнутых множеств замкнуто, пересечение выпуклых множеств выпукло, поэтому $N_{18}$ - замкнутый выпуклый многогранник. В силу леммы 1 , биективности отображения $\phi_{18}$ и в силу того, что $\mu_{j}\left(C_{k}^{0}\right)=\sum_{\sigma} \mu_{j}\left(C_{k}^{0} \cap B_{\sigma}\right)=\sum_{\sigma} x_{\sigma}^{(j), k}$, выполнены следующие равенства:

$\bar{R}=\sup _{\vec{\mu} \in \Lambda_{18}} f\left(\vec{\mu}\left(\vec{C}^{0}\right)\right)=\sup _{\vec{\mu} \in \Lambda_{18}} f\left(\left\{\mu_{j}\left(C_{k}^{0}\right)\right\}_{j, k=1}^{3}\right)=\sup _{\vec{x} \in N_{18}} f\left(\left\{\sum_{\sigma \in S_{3}} x_{\sigma}^{(j), k}\right\}_{j, k=1}^{3}\right)$.

Таким образом, формула для величины $\bar{R}$ из теоремы 2 доказана, и задача о нахождении величины $\bar{R}$ свелась к задаче о поиске экстремума на подмножестве множества $[0,1]^{54}$.

Теперь перейдем к вопросу о нахождении $\underline{R}$. Рассмотрим пространство $S_{3}=\{123,132,213,231,312,321\}$ и $\sigma$-алгебру $2^{S_{3}}$ на нем. Через $Z_{6}$ обозначим семейство $\vec{r}$-согласованных троек мер $\mu_{1}, \mu_{2}, \mu_{3}$ на $S_{3}$, т.е. $Z_{6}=\left\{\left\{\mu_{i}\right\}_{i=1}^{3}: \rho\left(\mu_{s}, \mu_{l}\right)=r_{s l}, 1 \leqslant s<l \leqslant 3\right\}$. Рассмотрим (по аналогии с введением множеств $B_{i j k}$ ) те тройки мер из $Z_{6}$, для которых при любом $\sigma \in S_{3}$ выполнены следующие соотношения:

$$
\mu_{\sigma_{1}}(\sigma) \geqslant \mu_{\sigma_{2}}(\sigma) \geqslant \mu_{\sigma_{3}}(\sigma) .
$$

Множество таких троек мер обозначим $\Lambda_{6}$. Напомним, что по определению $\underline{R}=\inf _{\vec{\mu} \in D(\vec{r})} \sup _{\vec{C} \in \mathscr{C}(\Omega)} f(\vec{\mu}(\vec{C}))$. Верна следующая лемма.

Лемма 2. Пусть в б-алгебре измеримого пространства $(\Omega, \Sigma)$ содержатся по крайней мере 6 непустых попарно не пересекаюшихся множеств $K_{\sigma}, \sigma \in S_{3}$. Тогда для любой функиии $f:[0,1]^{9} \rightarrow \mathbf{R}$ вьлолняется следующее равенство:

$$
\underline{R}=\inf _{\vec{\mu} \in \Lambda_{6}} \max _{\vec{C} \in \mathscr{C}\left(S_{3}\right)} f(\vec{\mu}(\vec{C})) .
$$

Д о к а з а т е л ь с т о о. Докажем сначала, что

$$
\underline{R} \leqslant \inf _{\vec{\mu} \in \Lambda_{6}} \sup _{\vec{C} \in \mathscr{C}\left(S_{3}\right)} f(\vec{\mu}(\vec{C})) .
$$

Так как $K_{\sigma} \neq \varnothing, \sigma \in S_{3}$, то существуют такие элементы $v_{\sigma}$, что $v_{\sigma} \in K_{\sigma}$, $\sigma \in S_{3}$. Для любой $\vec{r}$-согласованной тройки $\vec{\mu}$ мер на $S_{3}$ построим 
соответствующую тройку $\vec{\mu}^{*}$ мер на $\Omega$ следующим образом: $\mu_{i}^{*}(A)=$ $\sum_{\sigma: v_{\sigma} \in A} \mu_{i}(\sigma)$ при любом $A \in \Sigma$. Заметим, что $\sup _{\vec{C} \in \mathscr{C}\left(S_{3}\right)} f(\vec{\mu}(\vec{C}))=$ $\sup _{\vec{C} \in \mathscr{C}(\Omega)} f\left(\vec{\mu}^{*}(\vec{C})\right)$ и $\vec{\mu}^{*} \in D(\vec{r})$. Другими словами, для любой $\vec{r}-$ согласованной тройки $\vec{\mu}$ мер на $S_{3}$ найдется такая $\vec{r}$-согласованная тройка $\vec{\mu}^{*}$ мер на $\Omega$, что $\sup _{\vec{C} \in \mathscr{C}\left(S_{3}\right)} f(\vec{\mu}(\vec{C}))=\sup _{\vec{C} \in \mathscr{C}(\Omega)} f\left(\vec{\mu}^{*}(\vec{C})\right)$. Значит, $\inf _{\vec{\mu} \in D(\vec{r})} \sup _{\vec{C} \in \mathscr{C}(\Omega)} f(\vec{\mu}(\vec{C})) \leqslant \inf _{\vec{\mu} \in Z_{6}} \sup _{\vec{C} \in \mathscr{C}\left(S_{3}\right)} f(\vec{\mu}(\vec{C}))$. Далее, $\inf _{\vec{\mu} \in Z_{6}} \sup _{\vec{C} \in \mathscr{C}\left(S_{3}\right)} f(\vec{\mu}(\vec{C})) \leqslant \inf _{\vec{\mu} \in \Lambda_{6}} \sup _{\vec{C} \in \mathscr{C}\left(S_{3}\right)} f(\vec{\mu}(\vec{C}))$, так как $\Lambda_{6} \subset Z_{6}$. Таким образом,

$$
\inf _{\vec{\mu} \in D(\vec{r})} \sup _{\vec{C} \in \mathscr{C}(\Omega)} f(\vec{\mu}(\vec{C})) \leqslant \inf _{\vec{\mu} \in \Lambda_{6}} \sup _{\vec{C} \in \mathscr{C}\left(S_{3}\right)} f(\vec{\mu}(\vec{C}))
$$

причем в левой части последнего неравенства стоит не что иное, как величина $\underline{R}$.

Покажем теперь, что $\underline{R} \geqslant \inf _{\vec{\mu} \in \Lambda_{6}} \sup _{\vec{C} \in \mathscr{C}\left(S_{3}\right)} f(\vec{\mu}(\vec{C}))$. В самом деле, пусть $\vec{\mu}=\left(\mu_{1}, \mu_{2}, \mu_{3}\right)$ — тройка мер на измеримом пространстве $(\Omega, \Sigma)$. Для этой тройки мер определим множества $B_{\sigma}$ так же, как это было сделано выше (с помощью теоремы Хана о разложении). Далее, рассмотрим такие множества $C_{m}$, что $\bigsqcup_{m=1}^{3} C_{m}=\Omega$ и каждое $C_{m}$ является объединением нескольких множеств $B_{\sigma}$ (или же является пустым). Класс таких разбиений пространства $\Omega$ обозначим Г. Очевидно, что $\sup _{\vec{C} \in \mathscr{C}(\Omega)} f(\vec{\mu}(\vec{C})) \geqslant \sup _{\vec{C} \in \Gamma} f(\vec{\mu}(\vec{C}))$. Далее, рассмотрим меры $\mu_{k}^{*}(1 \leqslant k \leqslant 3)$ на $S_{3}$, определенные так: $\mu_{k}^{*}(\sigma)=\mu_{k}\left(B_{\sigma}\right)$. Несложно видеть, что определенные таким образом $\mu_{k}^{*}, 1 \leqslant k \leqslant 3$, будут $\vec{r}$ согласованными (так как $\left\{\mu_{i}\right\}_{i=1}^{3}$ являются $\vec{r}$-согласованными, а условия на $r_{i j}$ дают ограничения не более, чем на соотношения между мерами на множествах $\left.B_{i j k}\right)$. Кроме того, непосредственной проверкой можно убедиться, что $\sup _{\vec{C} \in \Gamma} f(\vec{\mu}(\vec{C}))=\sup _{\vec{C} \in \mathscr{C}\left(S_{3}\right)} f\left(\vec{\mu}^{*}(\vec{C})\right)$. Отсюда следует, что $\sup _{\vec{C} \in \mathscr{C}(\Omega)} f(\vec{\mu}(\vec{C})) \geqslant \sup _{\vec{C} \in \mathscr{C}\left(S_{3}\right)} f\left(\vec{\mu}^{*}(\vec{C})\right)$. Далее, покажем, что $\vec{\mu}^{*} \in \Lambda_{6}$, проверив выполнение условия (6) для тройки мер $\vec{\mu}^{*}$. В самом деле, в силу определения множеств $B_{\sigma}$ выполняются следующие неравенства: $\mu_{\sigma_{1}}\left(B_{\sigma}\right) \geqslant \mu_{\sigma_{2}}\left(B_{\sigma}\right) \geqslant \mu_{\sigma_{3}}\left(B_{\sigma}\right)$, поэтому $\mu_{\sigma_{1}}^{*}(\sigma) \geqslant \mu_{\sigma_{2}}^{*}(\sigma) \geqslant \mu_{\sigma_{3}}^{*}(\sigma)$. Значит, $\vec{\mu}^{*} \in \Lambda_{6}$. Таким образом, для любой $\vec{r}$-согласованной тройки $\vec{\mu}$ мер на $\Omega$ найдется такая $\vec{r}$-согласованная тройка $\vec{\mu}^{*}$ мер (из $\Lambda_{6}$ ) на $S_{3}$, что $\sup _{\vec{C} \in \mathscr{C}(\Omega)} f(\vec{\mu}(\vec{C})) \geqslant \sup _{\vec{C} \in \mathscr{C}\left(S_{3}\right)} f\left(\vec{\mu}^{*}(\vec{C})\right)$. Значит,

$$
\inf _{\vec{\mu} \in D(\vec{r})} \sup _{\vec{C} \in \mathscr{C}(\Omega)} f(\vec{\mu}(\vec{C})) \geqslant \inf _{\vec{\mu} \in \Lambda_{6}} \sup _{\vec{C} \in \mathscr{C}\left(S_{3}\right)} f(\vec{\mu}(\vec{C}))
$$

причем в левой части последнего неравенства стоит не что иное, как величина $\underline{R}$. Таким образом, $\underline{R} \geqslant \inf _{\vec{\mu} \in \Lambda_{6}} \sup _{\vec{C} \in \mathscr{C}\left(S_{3}\right)} f(\vec{\mu}(\vec{C}))$. Значит, $\underline{R}=\inf _{\vec{\mu} \in \Lambda_{6}} \sup _{\vec{C} \in \mathscr{C}\left(S_{3}\right)} f(\vec{\mu}(\vec{C}))$. Так как $S_{3}$ конечно, то sup и $\max$ по $\mathscr{C}\left(S_{3}\right)$ совпадают, что завершает доказательство леммы 2. 
Заметим, что лемма 2 существенно упрощает задачу о нахождении числа $\underline{R}$ : можно считать, что $\Omega=S_{3}, \Sigma=2^{S_{3}}$ и что тройка мер $\left(\mu_{1}, \mu_{2}, \mu_{3}\right)$ принадлежит множеству $\Lambda_{6}$.

Теперь докажем формулу для величины $\underline{R}$ из теоремы 2, т.е. сведем задачу о поиске $\underline{R}$ к поиску экстремума в $\mathbf{R}^{18}$ (предполагая, что условия теоремы 2 выполнены). Рассмотрим множество $\mathbb{T}$ троек произвольных отображений $T_{i}, T_{i}: S_{3} \rightarrow[0,1], 1 \leqslant i \leqslant 3$. Пусть $\phi_{6}: \mathbb{T} \rightarrow[0,1]^{18}$ и $\phi_{6}\left(T_{1}, T_{2}, T_{3}\right)=\vec{x}$, где $\vec{x}=\left(x_{123}^{(1)}, x_{132}^{(1)}, x_{213}^{(1)}, x_{231}^{(1)}, x_{312}^{(1)}\right.$, $\left.x_{321}^{(1)}, x_{123}^{(2)}, x_{132}^{(2)}, x_{213}^{(2)}, x_{231}^{(2)}, x_{312}^{(2)}, x_{321}^{(2)}, x_{123}^{(3)}, x_{132}^{(3)}, x_{213}^{(3)}, x_{231}^{(3)}, x_{312}^{(3)}, x_{321}^{(3)}\right)$, где, в свою очередь, $x_{i j k}^{(l)}=T_{l}\left(\omega_{i j k}\right)$. Несложно видеть, что такое отображение является биекцией между $\mathbb{T}$ и $[0,1]^{18}$.

Найдем образ множества $\Lambda_{6}$ при отображении $\phi_{6}$. Для этого фиксируем тройку мер $\left\{\mu_{i}\right\}_{i=1}^{3} \in \Lambda_{6}$. Тогда $x_{\sigma}^{(i)}=\mu_{i}(\sigma)$, где $1 \leqslant i \leqslant 3$, $\sigma=\left(\sigma_{1} \sigma_{2} \sigma_{3}\right) \in S_{3}$. Далее, расписывая $r_{i j}$ по определению, получаем

$$
2 r_{i j}=\sum_{\sigma \in S_{3}}\left(x_{\sigma}^{(i)}-x_{\sigma}^{(j)}\right)(-1)^{I\left\{\sigma_{i}^{-1}>\sigma_{j}^{-1}\right\}}, \quad 1 \leqslant i<j \leqslant 3 .
$$

Несложно видеть, что так как $\vec{\mu} \in \Lambda_{6}$, то

$$
x_{\sigma}^{\left(\sigma_{1}\right)} \geqslant x_{\sigma}^{\left(\sigma_{2}\right)} \geqslant x_{\sigma}^{\left(\sigma_{3}\right)} .
$$

Кроме того, в силу того, что $\mu_{i}, 1 \leqslant i \leqslant 3$, являются вероятностными мерами, выполняются следующие соотношения:

$$
\sum_{\sigma \in S_{3}} x_{\sigma}^{(l)}=1 \quad\left(0 \leqslant x_{\sigma}^{(l)} \leqslant 1,1 \leqslant l \leqslant 3, \sigma \in S_{3}\right) .
$$

Обозначим через $N_{6} \subset[0,1]^{18}$ множество таких векторов $\vec{x}=$ $\left(x_{123}^{(1)}, x_{132}^{(1)}, x_{213}^{(1)}, x_{231}^{(1)}, x_{312}^{(1)}, x_{321}^{(1)}, x_{123}^{(2)}, x_{132}^{(2)}, x_{213}^{(2)}, x_{231}^{(2)}, x_{312}^{(2)}, x_{321}^{(2)}, x_{123}^{(3)}, x_{132}^{(3)}, x_{213}^{(3)}\right.$, $\left.x_{231}^{(3)}, x_{312}^{(3)}, x_{321}^{(3)}\right)$, для которых выполнены условия $(7)-(9)$. Далее, множество $N_{6}$ представимо в виде конечного пересечения (замкнутых) полупространств и гиперплоскостей, пересечение замкнутых множеств замкнуто, пересечение выпуклых множеств выпукло, поэтому $N_{6}$ - замкнутый выпуклый многогранник. Заметим, что отображение $\phi_{6}$ устанавливает биекцию между $\Lambda_{6}$ и множеством $N_{6}$, так как условие принадлежности тройки мер множеству $\Lambda_{6}$ равносильно условиям $(7)-(9)$ принадлежности вектора $\vec{x}$ множеству $N_{6}$. В силу леммы 2 выполнено следующее равенство:

$$
\underline{R}=\inf _{\vec{\mu} \in \Lambda_{6}} \max _{\vec{C} \in \mathscr{C}\left(S_{3}\right)} f(\vec{\mu}(\vec{C})) .
$$

Напомним, что через $\mathscr{C}\left(S_{3}\right)$ мы обозначили множество всевозможных разбиений $S_{3}$ на 3 непересекающихся множества, т.е. разбиений вида 
$\vec{C}=\left\{C_{1}, C_{2}, C_{3}\right\}$, где $\bigsqcup_{k=1}^{3} C_{k}=S_{3}$. Так как $\phi_{6}$ - биекция и так как $\mu_{l}(\sigma)=x_{\sigma}^{(l)}, 1 \leqslant l \leqslant 3, \sigma \in S_{3}$, получаем

$$
\begin{aligned}
\underline{R}=\inf _{\vec{\mu} \in \Lambda_{6}} \max _{\vec{C} \in \mathscr{C}\left(S_{3}\right)} f(\vec{\mu}(\vec{C})) & =\inf _{\vec{\mu} \in \Lambda_{6}} \max _{\vec{C} \in \mathscr{C}\left(S_{3}\right)} f\left(\left\{\mu_{j}\left(C_{k}\right)\right\}_{j, k=1}^{3}\right) \\
& =\inf _{\vec{x} \in N_{6}} \max _{\vec{C} \in \mathscr{C}\left(S_{3}\right)} f\left(\left\{\sum_{\sigma \in C_{k}} x_{\sigma}^{(j)}\right\}_{j, k=1}^{3}\right) .
\end{aligned}
$$

Таким образом, теорема 2 доказана, а задача о нахождении величины $\underline{R}$ свелась к задаче о поиске экстремума на подмножестве множества $[0,1]^{18}$.

4. Доказательство теоремы 3. Перейдем к получению формулы для величины $\bar{R}$ из теоремы 3 . В силу теоремы 2 выполнено следующее соотношение: $\bar{R}=\sup _{\vec{x} \in N_{18}} f\left(\left\{\sum_{\sigma \in S_{3}} x_{\sigma}^{(j), k}\right\}_{j, k=1}^{3}\right)$. Рассмотрим линейное преобразование пространства $\mathbf{R}^{54} \rightarrow \mathbf{R}^{9}$, при котором каждому вектору $\vec{x} \in \mathbf{R}^{54}$ сопоставляется вектор $\vec{y}=\left(y_{1}, \ldots, y_{9}\right)$ так, что $y_{1}=\sum_{\sigma \in S_{3}} x_{\sigma}^{(1), 1}$, $y_{2}=\sum_{\sigma \in S_{3}} x_{\sigma}^{(1), 2}, \ldots, y_{9}=\sum_{\sigma \in S_{3}} x_{\sigma}^{(3), 3}$. Так как $N_{18}$ - выпуклый замкнутый многогранник в $\mathbf{R}^{54}$, то его образ $P$ при данном отображении тоже является выпуклым замкнутым многогранником. Тогда

$$
\bar{R}=\sup _{\vec{x} \in N_{18}} f\left(\left\{\sum_{\sigma \in S_{3}} x_{\sigma}^{(j), k}\right\}_{j, k=1}^{3}\right)=\sup _{\vec{y} \in P} f\left(y_{1}, \ldots, y_{9}\right) .
$$

Кроме того, если $\vec{x} \in N_{18}$, то $\sum_{j=1}^{3} \sum_{\sigma \in S_{3}} x_{\sigma}^{(i), j}=1, i=1,2,3$, поэтому если $\vec{y} \in P$, то $y_{1}+y_{2}+y_{3}=1, y_{4}+y_{5}+y_{6}=1$ и $y_{7}+y_{8}+y_{9}=1$. Пусть $Q_{6} \subset \mathbf{R}^{6}$ - проекция $P$ на подпространство пространства $\mathbf{R}^{9}$, порожденное координатами под номерами $1,2,4,5,7$ и 8 . Тогда $Q_{6}$ - выпуклый замкнутый многогранник. При любом $\vec{z}=\left(z_{1}, z_{2}, z_{3}, z_{4}, z_{5}, z_{6}\right) \in Q_{6}$ положим $f^{*}\left(z_{1}, z_{2}, z_{3}, z_{4}, z_{5}, z_{6}\right):=f\left(z_{1}, z_{2}, 1-z_{1}-z_{2}, z_{3}, z_{4}, 1-z_{3}-z_{4}, z_{5}, z_{6}, 1-\right.$ $\left.z_{5}-z_{6}\right)$, тогда $\bar{R}=\sup _{\vec{z} \in Q_{6}} f^{*}\left(z_{1}, z_{2}, z_{3}, z_{4}, z_{5}, z_{6}\right)$. Отметим, что множество $Q_{6}$ (как и множество $P$ ) строится независимо от функции $f$ (другими словами, множество $Q_{6}$ одинаково для всех функций $\left.f\right)$. Итак, формула для $\bar{R}$ из теоремы 3 доказана.

Перейдем к доказательству формулы для $\underline{R}$ из теоремы 3 . В силу теоремы 2 имеем $\underline{R}=\inf _{\vec{x} \in N_{6}} \max _{\vec{C} \in \mathscr{C}\left(S_{3}\right)} f\left(\left\{\sum_{\sigma \in C_{k}} x_{\sigma}^{(j)}\right\}_{j, k=1}^{3}\right)$. Обозначим через $V_{1} \subset N_{6}$ такое (возможно, пустое) множество, что при всех $\vec{x} \in V_{1}$ выполнено соотношение $\max _{\vec{C} \in \mathscr{C}\left(S_{3}\right)} f\left(\left\{\sum_{\sigma \in C_{k}} x_{\sigma}^{(j)}\right\}_{j, k=1}^{3}\right)=$ $f\left(\left\{\sum_{\sigma \in C_{k}^{*}} x_{\sigma}^{(j)}\right\}_{j, k=1}^{3}\right)$, где $C_{1}^{*}=\{(123),(231),(312)\}, C_{2}^{*}=\{(132),(321)\}$, $C_{3}^{*}=\{(213)\}$. Рассмотрим (вырожденное) линейное отображение $\beta_{1}$ : $\mathbf{R}^{18} \rightarrow \mathbf{R}^{9}$, при котором каждому вектору $\vec{x} \in N_{6}$ сопоставляется вектор 
$\vec{y} \in \mathbf{R}^{9}$ так, что

$$
\begin{array}{lll}
y_{1}=x_{123}^{1}+x_{231}^{1}+x_{312}^{1}, & y_{2}=x_{132}^{1}+x_{321}^{1}, & y_{3}=x_{213}^{1}, \\
y_{4}=x_{123}^{2}+x_{231}^{2}+x_{312}^{2}, & y_{5}=x_{132}^{2}+x_{321}^{2}, & y_{6}=x_{213}^{2}, \\
y_{7}=x_{123}^{3}+x_{231}^{3}+x_{312}^{3}, & y_{8}=x_{132}^{3}+x_{321}^{3}, & y_{9}=x_{213}^{3}
\end{array}
$$

Обозначим через $U_{1} \subset \mathbf{R}^{9}$ образ $V_{1}$ при отображении $\beta_{1}$. Рассматривая всевозможные разбиения $S_{3}$ на 3 подмножества (число таких разбиений обозначим через $T$ ) получаем набор $\left\{V_{i}\right\}_{i=1}^{T}$ таких множеств, что $V_{i} \subset N_{6}$ при $1 \leqslant i \leqslant T, \bigcup_{i=1}^{T} V_{i}=N_{6}$ и для каждого $V_{i}, 1 \leqslant i \leqslant T$, определено соответствующее (вырожденное) линейное преобразование $\beta_{i}$. Образ $V_{i}$ при отображении $\beta_{i}$ обозначим через $U_{i}$. Пусть $F:=\bigcup_{i=1}^{T} U_{i}$. Тогда $F \subset \mathbf{R}^{9}$ и

$$
\begin{aligned}
\underline{R} & =\inf _{\vec{x} \in N_{6}} \max _{\vec{C} \in \mathscr{C}\left(S_{3}\right)} f\left(\left\{\sum_{\sigma \in C_{k}} x_{\sigma}^{(j)}\right\}_{j, k=1}^{3}\right) \\
& =\inf _{\vec{x} \in \bigcup_{i=1}^{T} \max _{V_{i}} \vec{C} \in \mathscr{C}\left(S_{3}\right)} f\left(\left\{\sum_{\sigma \in C_{k}} x_{\sigma}^{(j)}\right\}_{j, k=1}^{3}\right) \\
& =\min _{1 \leqslant i \leqslant T} \inf _{\vec{x} \in V_{i}} \max _{\vec{C} \in \mathscr{C}\left(S_{3}\right)} f\left(\left\{\sum_{\sigma \in C_{k}} x_{\sigma}^{(j)}\right\}_{j, k=1}^{3}\right) \\
& =\min _{1 \leqslant i \leqslant T} \inf _{\vec{y} \in U_{i}} f\left(y_{1}, y_{2}, \ldots, y_{9}\right) \\
& =\inf _{\vec{y} \in \bigcup_{i=1}^{T} U_{i}} f\left(y_{1}, y_{2}, \ldots, y_{9}\right) \\
& =\inf _{\vec{y} \in F} f\left(y_{1}, y_{2}, \ldots, y_{9}\right) .
\end{aligned}
$$

Отметим, что (как и ранее) если $\vec{y} \in F$, то $y_{1}+y_{2}+y_{3}=1, y_{4}+y_{5}+y_{6}=1$ и $y_{7}+y_{8}+y_{9}=1$. Далее, пусть $G_{6} \subset \mathbf{R}^{6}-$ проекция $F$ на подпространство пространства $\mathbf{R}^{9}$, порожденное координатами под номерами $1,2,4,5$, 7, 8. При любом $\vec{z}=\left(z_{1}, z_{2}, z_{3}, z_{4}, z_{5}, z_{6}\right) \in G_{6}$ положим (как и ранее) $f^{*}\left(z_{1}, z_{2}, z_{3}, z_{4}, z_{5}, z_{6}\right):=f\left(z_{1}, z_{2}, 1-z_{1}-z_{2}, z_{3}, z_{4}, 1-z_{3}-z_{4}, z_{5}, z_{6}, 1-z_{5}-z_{6}\right)$, тогда $\underline{R}=\inf _{\vec{z} \in G_{6}} f^{*}\left(z_{1}, z_{2}, z_{3}, z_{4}, z_{5}, z_{6}\right)$. Теорема 3 доказана.

Отметим, что в отличие от множества $Q_{6}$ множество $G_{6}$ зависит от функции $f$, и в этом смысле формула, сводящая задачу о нахождении $\underline{R}$ к поиску экстремума на $G_{6}$, менее удобна, чем соответствующая формула для $\bar{R}$ из теоремы 3 . Более того, в отличие от множества $P_{6}$, которое является замкнутым выпуклым многогранником, множество $G_{6}$, вообще говоря, устроено значительно сложнее.

Отметим также, что формулы для величины $\underline{R}$ в теоремах 2 и 3 останутся в силе, если в $\sigma$-алгебре измеримого пространства $(\Omega, \Sigma)$ содержится по крайней мере 6 непустых попарно не пересекающихся множеств. 
5. Доказательство теоремы 1. Теперь получим формулу для величины $\underline{R}$, которая потребуется для доказательства теоремы 1 , т.е. для нахождения величин $\underline{R}^{+}$и $\bar{R}^{+}$. Верна следующая лемма.

Лемма 3. Пусть в б-алгебре измеримого пространства $(\Omega, \Sigma)$ содержится по крайней мере 18 непустьх попарно не пересекающихся множеств. Тогда для любой функиии $f$, определенной на множестве $[0,1]^{9}$, выполнены следуюшие равенства:

$$
\underline{R}=\inf _{\vec{\mu} \in \Lambda_{18}} \sup _{\vec{C} \in \mathscr{C}\left(\Omega_{18}\right)} f(\vec{\mu}(\vec{C})), \quad \bar{R}=\sup _{\vec{\mu} \in \Lambda_{18}} \sup _{\vec{C} \in \mathscr{C}\left(\Omega_{18}\right)} f(\vec{\mu}(\vec{C})) .
$$

Д о к а з а т е л ь с т в о. Несложно видеть, что

$$
\underline{R} \leqslant \inf _{\vec{\mu} \in \Lambda_{18}} \sup _{\vec{C} \in \mathscr{C}\left(\Omega_{18}\right)} f(\vec{\mu}(\vec{C}))
$$

(в силу тех же соображений, с помощью которых в лемме 2 устанавливается неравенство $\underline{R} \leqslant \inf _{\vec{\mu} \in \Lambda_{6}} \sup _{\vec{C} \in \mathscr{C}\left(S_{3}\right)} f(\vec{\mu}(\vec{C}))$. Далее, без ограничения общности можно считать, что $S_{3} \subset \Omega_{18}$, поэтому $\Lambda_{6} \subset \Lambda_{18}$. В силу леммы 2 и конечности $S_{3}$ получаем, что

$$
\begin{aligned}
\underline{R}=\inf _{\vec{\mu} \in \Lambda_{6}} \max _{\vec{C} \in \mathscr{C}\left(S_{3}\right)} f(\vec{\mu}(\vec{C})) & =\inf _{\vec{\mu} \in \Lambda_{6}} \sup _{\vec{C} \in \mathscr{C}\left(S_{3}\right)} f(\vec{\mu}(\vec{C})) \\
& \geqslant \inf _{\vec{\mu} \in \Lambda_{18}} \sup _{\vec{C} \in \mathscr{C}\left(\Omega_{18}\right)} f(\vec{\mu}(\vec{C})) \geqslant \underline{R} .
\end{aligned}
$$

Таким образом, $\underline{R}=\inf _{\vec{\mu} \in \Lambda_{18}} \sup _{\vec{C} \in \mathscr{C}\left(\Omega_{18}\right)} f(\vec{\mu}(\vec{C}))$. Аналогично с помощью леммы 1 получаем, что

$$
\begin{aligned}
\bar{R}=\sup _{\vec{\mu} \in \Lambda_{18}} f\left(\vec{\mu}\left(\vec{C}^{0}\right)\right) & \leqslant \sup _{\vec{\mu} \in \Lambda_{18}} \sup _{\vec{C} \in \mathscr{C}\left(\Omega_{18}\right)} f(\vec{\mu}(\vec{C})) \\
& \leqslant \sup _{\vec{\mu} \in D(\vec{r})} \sup _{\vec{C} \in \mathscr{C}(\Omega)} f(\vec{\mu}(\vec{C}))=\bar{R},
\end{aligned}
$$

поэтому $\bar{R}=\sup _{\vec{\mu} \in \Lambda_{18}} \sup _{\vec{C} \in \mathscr{C}\left(\Omega_{18}\right)} f(\vec{\mu}(\vec{C}))$, что завершает доказательство леммы 3.

Далее, упомянем результат, который не будет использоваться в дальнейшем, хотя и представляет определенный интерес.

3 а м е ч а н и е 1 . Пусть выполнены условия теоремы 1 и функция $f$ непрерывна. Тогда для любого числа $а$ такого, что $a \in[\underline{R}, \bar{R}]$, существует такая тройка мер $\vec{\mu} \in \Lambda_{18}$, что $\max _{\vec{C} \in \mathscr{C}\left(\Omega_{18}\right)} f(\vec{\mu}(\vec{C}))=a$.

Далее, для доказательства теоремы 1 нам потребуется следующее вспомогательное утверждение.

Лемма 4. Пусть $\Omega$ не более, чем счетно, $\Sigma=2^{\Omega}$, пусть $\bigsqcup_{i=1}^{3} C_{i}^{\mathrm{opt}}=\Omega$ ина множестве $C_{i}^{\mathrm{opt}}$ мера $\mu_{i}$ не меньше других мер, т.е. 
для любого $\omega \in C_{i}^{\mathrm{opt}}$ вьлолняется соотношение $\mu_{i}(\omega) \geqslant \max _{j} \mu_{j}(\omega)$. Тогда

$$
\sup _{\vec{C} \in \mathscr{C}(\Omega)} \sum_{i=1}^{3} \mu_{i}\left(C_{i}\right)=\sum_{i=1}^{3} \mu_{i}\left(C_{i}^{\mathrm{opt}}\right)
$$

Д о к а з а т е л с с в о. Достаточно доказать, что для любого разбиения $C_{i}, 1 \leqslant i \leqslant 3, \sqcup C_{i}=\Omega$, справедливо неравенство $\sum_{i=1}^{3} \mu_{i}\left(C_{i}\right) \leqslant$ $\sum_{i=1}^{3} \mu_{i}\left(C_{i}^{\text {opt }}\right)$. Положим $D_{i j}:=C_{i}^{\text {opt }} \cap C_{j}, x_{i j}^{k}:=\mu_{k}\left(D_{i j}\right)$. Тогда

$$
\sum_{j=1}^{3} \mu_{j}\left(C_{j}\right)=\sum_{j=1}^{3} \sum_{i=1}^{3} \mu_{j}\left(D_{i j}\right)=\sum_{j=1}^{3} \sum_{i=1}^{3} x_{i j}^{j} \leqslant \sum_{i=1}^{3} \sum_{j=1}^{3} x_{i j}^{i},
$$

так как из определения $C_{i}^{\text {opt }}$ следует, что $x_{i j}^{i} \geqslant x_{i j}^{j}$. Далее,

$$
\sum_{i=1}^{3} \mu_{i}\left(C_{i}^{\mathrm{opt}}\right)=\sum_{i=1}^{3} \sum_{j=1}^{3} \mu_{i}\left(D_{i j}\right)=\sum_{i=1}^{3} \sum_{j=1}^{3} x_{i j}^{i}
$$

поэтому $\sum_{i=1}^{3} \mu_{i}\left(C_{i}\right) \leqslant \sum_{i=1}^{3} \mu_{i}\left(C_{i}^{\text {opt }}\right)$, что завершает доказательство леммы 4.

Д о к а з а т е л ь с т в о. Докажем, что для любых $C_{j}, 1 \leqslant j \leqslant 3$, таких, что $\sqcup C_{j}=\Omega$ следует, что $\sum_{j=1}^{3} \mu_{j}\left(C_{j}\right) \leqslant \sum_{j=1}^{3} \mu_{j}\left(C_{j}^{00}\right)$. Введем $D_{i j}=C_{i}^{\mathrm{opt}} \cap C_{j}, \mu_{k}\left(D_{i j}\right)=x_{i j}^{k}$. Тогда $\sum_{j=1}^{3} \mu_{j}\left(C_{j}\right)=\sum_{j=1}^{3} \sum_{i=1}^{3} \mu_{j}\left(D_{i j}\right)=$ $\sum_{j=1}^{3} \sum_{i=1}^{3} x_{i j}^{j} \leqslant \sum_{j=1}^{3} \sum_{i=1}^{3} x_{i j}^{i}$ так как из определения $C_{i}^{\text {opt }}$ следует, что $x_{i j}^{i} \geqslant x_{i j}^{j}$. Но $\sum_{i=1}^{3} \mu_{i}\left(C_{i}^{\text {opt }}\right)=\sum_{i=1}^{3} \sum_{j=1}^{3} \mu_{i}\left(D_{i j}\right)=\sum_{i=1}^{3} \sum_{j=1}^{3} x_{i j}^{i}$, что завершает доказательство леммы 4.

Теперь докажем теорему 1 , получив удобные формулы для величин

$$
\underline{R}^{+}=\inf _{\vec{\mu} \in D(\vec{r})} \sup _{\vec{C} \in \mathscr{C}(\Omega)} \sum_{i=1}^{3} \mu_{i}\left(C_{i}\right), \quad \bar{R}^{+}=\sup _{\vec{\mu} \in D(\vec{r})} \sup _{\vec{C} \in \mathscr{C}(\Omega)} \sum_{i=1}^{3} \mu_{i}\left(C_{i}\right) .
$$

Пусть выполнены условия теоремы 1. В силу леммы 3 в этом случае

$$
\underline{R}^{+}=\inf _{\vec{\mu} \in \Lambda_{18}} \sup _{\vec{C} \in \mathscr{C}\left(\Omega_{18}\right)} \sum_{i=1}^{3} \mu_{i}\left(C_{i}\right), \quad \bar{R}^{+}=\sup _{\vec{\mu} \in \Lambda_{18}} \sup _{\vec{C} \in \mathscr{C}\left(\Omega_{18}\right)} \sum_{i=1}^{3} \mu_{i}\left(C_{i}\right) .
$$

Заметим, что множества $C_{i}^{*}=\bigsqcup_{m=1}^{3}\left(\omega_{i j k}^{m} \sqcup \omega_{i k j}^{m}\right)$ (для $\left.(i j k) \in S_{3}\right)$ подходят в качестве $C_{i}^{\text {opt }}$ для любой тройки мер $\vec{\mu} \in \Lambda_{18}$ (в силу определения $\left.\Lambda_{18}\right)$. Например, для $\omega_{123}^{m}, 1 \leqslant m \leqslant 3$, имеем $\mu_{1}\left(w_{123}^{m}\right) \geqslant \mu_{2}\left(w_{123}^{m}\right) \geqslant$ $\mu_{3}\left(w_{123}^{m}\right)$.

Поэтому в силу леммы 4

$$
\begin{aligned}
& \underline{R}^{+}=\inf _{\vec{\mu} \in \Lambda_{18}} \sup _{\vec{C} \in \mathscr{C}\left(\Omega_{18}\right)} \sum_{i=1}^{3} \mu_{i}\left(C_{i}\right)=\inf _{\vec{\mu} \in \Lambda_{18}} \sum_{i=1}^{3} \mu_{i}\left(C_{i}^{*}\right), \\
& \bar{R}^{+}=\sup _{\vec{\mu} \in \Lambda_{18}} \sup _{\vec{C} \in \mathscr{C}\left(\Omega_{18}\right)} \sum_{i=1}^{3} \mu_{i}\left(C_{i}\right)=\sup _{\vec{\mu} \in \Lambda_{18}} \sum_{i=1}^{3} \mu_{i}\left(C_{i}^{*}\right) .
\end{aligned}
$$


В силу биективности отображения $\phi_{18}$, используя обозначения для векторов из $N_{18}$, получаем

$$
\begin{aligned}
& \underline{R}^{+}=\inf _{\vec{\mu} \in \Lambda_{18}} \sum_{i=1}^{3} \mu_{i}\left(C_{i}^{*}\right)=\inf _{\vec{x} \in N_{18}} \sum_{i=1}^{3}\left(\sum_{m=1}^{3}\left(x_{i j k}^{(i), m}+x_{i k j}^{(i), m}\right)\right), \\
& \bar{R}^{+}=\sup _{\vec{\mu} \in \Lambda_{18}} \sum_{i=1}^{3} \mu_{i}\left(C_{i}^{*}\right)=\sup _{\vec{x} \in N_{18}} \sum_{i=1}^{3}\left(\sum_{m=1}^{3}\left(x_{i j k}^{(i), m}+x_{i k j}^{(i), m}\right)\right)
\end{aligned}
$$

для $(i j k) \in S_{3}$. Пусть $x_{\sigma}^{(i)}:=\sum_{m} x_{\sigma}^{(i), m}$. Несложно проверить, что в новых обозначениях задача о нахождении $\underline{R}^{+}$и $\bar{R}^{+}$перепишется следующим образом:

$$
\underline{R}^{+}=\inf _{\vec{x} \in N_{6}} \sum_{i=1}^{3}\left(x_{i j k}^{(i)}+x_{i k j}^{(i)}\right), \quad \bar{R}^{+}=\sup _{\vec{x} \in N_{6}} \sum_{i=1}^{3}\left(x_{i j k}^{(i)}+x_{i k j}^{(i)}\right) .
$$

Напомним, что множество $N_{6}$ задается системой из соотношений $(7),(9)$, а также шестью парами неравенств:

$$
x_{\sigma}^{\left(\sigma_{1}\right)} \geqslant x_{\sigma}^{\left(\sigma_{2}\right)} \geqslant x_{\sigma}^{\left(\sigma_{3}\right)}, \quad \sigma \in S_{3} .
$$

Введем новые переменные $\varepsilon_{i j k}(\vec{x}), \delta_{i j k}(\vec{x}), t_{i j k}(\vec{x})$ следующим образом: $x_{\sigma}^{\left(\sigma_{1}\right)}=x_{\sigma}^{\left(\sigma_{2}\right)}+\delta_{\sigma}(\vec{x}), x_{\sigma}^{\left(\sigma_{2}\right)}=x_{\sigma}^{\left(\sigma_{3}\right)}+\varepsilon_{\sigma}(\vec{x}), x_{\sigma}^{\left(\sigma_{3}\right)}=t_{\sigma}(\vec{x}), \sigma \in S_{3}$, причем в дальнейшем вместо $\varepsilon_{i j k}(\vec{x}), \delta_{i j k}(\vec{x}), t_{i j k}(\vec{x})$ будем писать $\varepsilon_{i j k}, \delta_{i j k}, t_{i j k}$ соответственно. В новых переменных множество $N_{6}$ описывается условиями

$$
\begin{aligned}
& 2 r_{12}=\delta_{123}+\varepsilon_{132}+\delta_{132}+\varepsilon_{312}+\delta_{213}+\varepsilon_{231}+\delta_{231}+\varepsilon_{321}, \\
& 2 r_{13}=\varepsilon_{123}+\delta_{123}+\delta_{132}+\varepsilon_{213}+\delta_{312}+\varepsilon_{321}+\delta_{321}+\varepsilon_{231}, \\
& 2 r_{23}=\varepsilon_{213}+\delta_{213}+\delta_{231}+\varepsilon_{123}+\varepsilon_{312}+\delta_{312}+\delta_{321}+\varepsilon_{132}, \\
& 1=\sum_{\sigma \in S_{3}} t_{\sigma}+\varepsilon_{123}+\delta_{123}+\varepsilon_{132}+\delta_{132}+\varepsilon_{213}+\varepsilon_{312}, \\
& 1=\sum_{\sigma \in S_{3}} t_{\sigma}+\varepsilon_{123}+\varepsilon_{213}+\delta_{213}+\varepsilon_{231}+\delta_{231}+\varepsilon_{321}, \\
& 1=\sum_{\sigma \in S_{3}} t_{\sigma}+\varepsilon_{132}+\varepsilon_{231}+\varepsilon_{312}+\delta_{312}+\varepsilon_{321}+\delta_{321}, \\
& \varepsilon_{i j k} \geqslant 0, \delta_{i j k} \geqslant 0, t_{i j k} \geqslant 0 .
\end{aligned}
$$

При этом $\underline{R}^{+}=\min _{N_{6}}\left(\sum_{\sigma \in S_{3}}\left(t_{\sigma}+\varepsilon_{\sigma}+\delta_{\sigma}\right)\right)$. Положим $t=\sum_{\sigma \in S_{3}} t_{\sigma}$, $\varepsilon=\sum_{\sigma} \varepsilon_{\sigma}, \delta=\sum_{\sigma} \delta_{\sigma}$. Сложив (10)-(12), а также (13)-(15), получим $2 \varepsilon+2 \delta=2\left(r_{12}+r_{13}+r_{23}\right)$ и $3 t+2 \varepsilon+\delta=3$ соответственно. Значит, $\underline{R}^{+}=\min _{N_{6}}(t+\varepsilon+\delta)$. Учитывая (16), получаем, что

$$
\begin{aligned}
1+\frac{1}{3}\left(r_{12}+r_{13}+r_{23}\right) & =\frac{1}{3}\left(3+r_{12}+r_{13}+r_{23}\right) \\
& =\frac{1}{3}(3 t+2 \varepsilon+\delta+\varepsilon+\delta) \leqslant t+\varepsilon+\delta .
\end{aligned}
$$


Кроме того,

$$
\begin{aligned}
t+\varepsilon+\delta & \leqslant \frac{1}{3}(3 t+2 \varepsilon+\delta+2(\varepsilon+\delta))=\frac{1}{3}\left(3+2\left(r_{12}+r_{13}+r_{23}\right)\right) \\
& =1+\frac{2}{3}\left(r_{12}+r_{13}+r_{23}\right) .
\end{aligned}
$$

Далее, несложно видеть, что $\bar{R}^{+}=\max _{N_{6}}(t+\varepsilon+\delta)$. Таким образом, нами доказано следующее предложение.

Предложение 1. При выполнении условий теоремы 1 верны следуюшие неравенства:

$$
1+\frac{1}{3}\left(r_{12}+r_{13}+r_{23}\right) \leqslant \underline{R}^{+} \leqslant \bar{R}^{+} \leqslant 1+\frac{2}{3}\left(r_{12}+r_{13}+r_{23}\right) .
$$

Далее, положим $\delta_{i}=\delta_{i k l}+\delta_{i l k}, \varepsilon_{i}=\varepsilon_{k l i}+\varepsilon_{l k i},(i k l) \in S_{3}$. Получаем, что теперь задача сводится к следующей: множество $K \subset \mathbf{R}^{7}$, состоящее из наборов чисел $\left(t, \delta_{1}, \delta_{2}, \delta_{3}, \varepsilon_{1}, \varepsilon_{2}, \varepsilon_{3}\right)$, задано следующими соотношениями:

$$
\begin{gathered}
\left(\delta_{k}+\varepsilon_{k}\right)+\left(\delta_{l}+\varepsilon_{l}\right)=2 r_{k l}, \quad k<l, \\
1=t+\delta_{i}+\varepsilon_{k}+\varepsilon_{l},(i k l) \in S_{3}, \quad k<l, \\
0 \leqslant t \leqslant 1, \quad 0 \leqslant \delta_{i} \leqslant 1, \quad 0 \leqslant \varepsilon_{i} \leqslant 1, \quad 1 \leqslant i \leqslant 3 .
\end{gathered}
$$

Нужно найти $\underline{R}^{+}=\min _{K}\left(t+\sum_{i=1}^{3}\left(\delta_{i}+\varepsilon_{i}\right)\right)$ и $\bar{R}^{+}=\max _{K}\left(t+\sum_{i=1}^{3}\left(\delta_{i}+\varepsilon_{i}\right)\right)$. Но $r_{12}+r_{13}+r_{23}=\left(\delta_{i}+\varepsilon_{i}\right)+\left(\delta_{k}+\varepsilon_{k}\right)+\left(\delta_{l}+\varepsilon_{l}\right)=2 r_{k l}+\left(\delta_{i}+\varepsilon_{i}\right)$. Значит, множество $K \subset \mathbf{R}^{7}$ задается условиями

$$
\begin{aligned}
\delta_{i}+\varepsilon_{i}= & r_{12}+r_{13}+r_{23}-2 r_{k l}=: r_{i}, \quad(i k l) \in S_{3}, \quad k<l, \\
1= & t+\delta_{i}+\varepsilon_{k}+\varepsilon_{l}, \quad(i k l) \in S_{3}, \quad k<l, \\
& 0 \leqslant t \leqslant 1, \quad 0 \leqslant \delta_{i} \leqslant 1, \quad 0 \leqslant \varepsilon_{i} \leqslant 1, \quad 1 \leqslant i \leqslant 3 .
\end{aligned}
$$

При этом в $(17) r_{i}=r_{12}+r_{13}+r_{23}-2 r_{k l} \geqslant 0$ по неравенству треугольника. Однако $\varepsilon+\delta=r_{12}+r_{13}+r_{23}$, поэтому $\underline{R}^{+}=r_{12}+r_{13}+r_{23}+\min _{K} t$ и, аналогично, $\bar{R}^{+}=r_{12}+r_{13}+r_{23}+\max _{K} t$.

Упростим задачу, выразив все через $\varepsilon_{i}, 1 \leqslant i \leqslant 3$. Из (18) следует, что $1+\varepsilon_{i}=1+t+\delta_{i}+\varepsilon_{k}+\varepsilon_{l}+\varepsilon_{i}$, поэтому $\varepsilon_{i}-\delta_{i}$ не зависит от $i$, т.е. $\delta_{1}-\varepsilon_{1}=\delta_{i}-\varepsilon_{i}, 1 \leqslant i \leqslant 3$. Но $\delta_{i}+\varepsilon_{i}=r_{i}$, поэтому $\delta_{i}=$ $\delta_{1}-\varepsilon_{1}+\varepsilon_{i}=\left(r_{1}-\varepsilon_{1}\right)-\varepsilon_{1}+\varepsilon_{i}$, значит, $\delta_{i}=r_{1}+\varepsilon_{i}-2 \varepsilon_{1}$. Поэтому $r_{i}=\delta_{i}+\varepsilon_{i}=\left(r_{1}+\varepsilon_{i}-2 \varepsilon_{1}\right)+\varepsilon_{i}$, откуда $\varepsilon_{i}=\varepsilon_{1}+\left(r_{i}-r_{1}\right) / 2$. Далее, учтем, что $\varepsilon_{i} \in[0,1]$ и что $\delta_{i}=r_{i}-\varepsilon_{i} \in[0,1]$. Тогда $0 \leqslant \varepsilon_{i} \leqslant \min \left(r_{i}, 1\right)$ и $\delta_{i} \leqslant 1$. При этом $t=1-\delta_{1}-\varepsilon_{2}-\varepsilon_{3}=1-\left(r_{1}-\varepsilon_{1}\right)-\varepsilon_{2}-\varepsilon_{3}$ (и в силу равенства $t=1-\delta_{1}-\varepsilon_{2}-\varepsilon_{3}$ получаем, что $t$ будет не больше единицы при неотрицательных $\delta_{i}$ и $\varepsilon_{i}$ ). Получаем, что теперь необходимо решить 
следующую задачу: $0 \leqslant \varepsilon_{i} \leqslant \min \left(r_{i}, 1\right), t \geqslant 0$,

$$
\begin{aligned}
& r_{1}+\varepsilon_{i}-2 \varepsilon_{1} \leqslant 1, \quad 1 \leqslant i \leqslant 3, \\
& \varepsilon_{i}=\varepsilon_{1}+\frac{r_{i}-r_{1}}{2}, \quad 1 \leqslant i \leqslant 3, \\
& t=1-r_{1}+\varepsilon_{1}-\varepsilon_{2}-\varepsilon_{3} \rightarrow \text { extr. }
\end{aligned}
$$

Выразим все через переменную $\varepsilon_{1}$ и сведем задачу к такой:

$$
\begin{aligned}
\frac{r_{1}-r_{i}}{2} \leqslant \varepsilon_{1} \leqslant & \frac{r_{1}+r_{i}}{2}, \quad \varepsilon_{1} \geqslant \frac{r_{1}+r_{i}}{2}-1, \quad \varepsilon_{1}+\frac{r_{i}-r_{1}}{2} \leqslant 1, \quad 1 \leqslant i \leqslant 3 \\
t & =1-r_{1}+\varepsilon_{1}-\left(\varepsilon_{1}+\frac{r_{2}-r_{1}}{2}+\varepsilon_{1}+\frac{r_{3}-r_{1}}{2}\right) \\
& =1-\frac{r_{2}+r_{3}}{2}-\varepsilon_{1} \rightarrow \text { extr, } \quad t \geqslant 0 .
\end{aligned}
$$

Подведем итог: для того, чтобы выполнялось условие $\vec{x} \in N_{6}$, необходимо, чтобы построенное по данному $\vec{x}$ число $\varepsilon_{1}$ удовлетворяло следующим условиям:

$$
\begin{gathered}
\max _{i} \max \left(\frac{r_{1}-r_{i}}{2}, \frac{r_{1}+r_{i}}{2}-1\right) \leqslant \varepsilon_{1}, \\
\varepsilon_{1} \leqslant \min _{i} \min \left(\frac{r_{1}+r_{i}}{2}, 1+\frac{r_{1}-r_{i}}{2}, 1-\frac{r_{2}+r_{3}}{2}\right) .
\end{gathered}
$$

И в рамках данных ограничений на $\varepsilon_{1}$ требуется найти экстремум выражения $1-\left(r_{2}+r_{3}\right) / 2-\varepsilon_{1} \rightarrow$ extr. Более того, несложно видеть (так как $t$ и все $\delta_{j}$ выражаются через $\varepsilon_{i}$, а все $\varepsilon_{i}$ выражаются через $\left.\varepsilon_{1}\right)$, что если $\varepsilon_{1}$, удовлетворяющее таким условиям, найдется, то по нему можно будет построить вектор из $N_{6}$.

Начиная с этого момента, без ограничения общности будем считать, что $r_{1} \geqslant r_{2} \geqslant r_{3}$ (это возможно, так как функционал $f$ инвариантен относительно перестановок мер). Другими словами, будем считать, что изначально меры были перенумерованы так, чтобы выполнялось соотношение $r_{1} \geqslant r_{2} \geqslant r_{3}$. Тогда (19) равносильно тому, что $\max \left(\left(r_{1}-r_{3}\right) / 2, r_{1}-\right.$ $1) \leqslant \varepsilon_{1} \leqslant \min \left(\frac{1}{2}\left(r_{1}+r_{3}\right), 1,1-\frac{1}{2}\left(r_{2}+r_{3}\right)\right)$. Однако $\frac{1}{2}\left(r_{1}+r_{3}\right)=r_{13} \leqslant 1$, поэтому $2-r_{3} \geqslant r_{1}$, откуда $r_{1}-r_{3} \geqslant 2 r_{1}-2$. Значит, $\frac{1}{2}\left(r_{1}-r_{3}\right) \geqslant r_{1}-1$. Кроме того, $r_{13}=\frac{1}{2}\left(r_{1}+r_{3}\right) \leqslant 1$, поэтому (19) равносильно тому, что $\varepsilon_{1} \in\left[\frac{1}{2}\left(r_{1}-r_{3}\right), \min \left(\frac{1}{2}\left(r_{1}+r_{3}\right), 1-\frac{1}{2}\left(r_{2}+r_{3}\right)\right)\right]$. Отметим, что данное множество непусто, так как $\frac{1}{2}\left(r_{1}-r_{3}\right) \leqslant 1-\frac{1}{2}\left(r_{2}+r_{3}\right)$ в силу того, что $r_{12}=\frac{1}{2}\left(r_{1}+r_{2}\right) \leqslant 1$. Ранее мы получили, что $\underline{R}^{+}=r_{12}+r_{13}+r_{23}+\min _{K} t$ и $\bar{R}^{+}=r_{12}+r_{13}+r_{23}+\max _{K} t$, при этом $t=1-\frac{1}{2}\left(r_{2}+r_{3}\right)-\varepsilon_{1}$, значит, $\bar{R}^{+}=r_{12}+r_{13}+r_{23}+1-\frac{1}{2}\left(r_{2}+r_{3}\right)-\frac{1}{2}\left(r_{1}-r_{3}\right)$ и, аналогично, $\underline{R}^{+}=r_{12}+r_{13}+r_{23}+1-\frac{1}{2}\left(r_{2}+r_{3}\right)-\min \left(\frac{1}{2}\left(r_{1}+r_{3}\right), 1-\frac{1}{2}\left(r_{2}+r_{3}\right)\right)$. 


$$
\begin{aligned}
& \text { Поэтому }\left(\text { так как }-\min _{Z} x=\max _{Z}(-x)\right) \\
& \begin{aligned}
\underline{R}^{+} & =r_{12}+r_{13}+r_{23}+1-\frac{r_{2}+r_{3}}{2}+\max \left(-\frac{r_{1}+r_{3}}{2}, \frac{r_{2}+r_{3}}{2}-1\right) \\
& =r_{12}+r_{13}+r_{23}+1-r_{23}+\max \left(-r_{13}, r_{23}-1\right) \\
& =\max \left(1+r_{12}, r_{12}+r_{13}+r_{23}\right) .
\end{aligned}
\end{aligned}
$$

Далее, так как $r_{1} \geqslant r_{2} \geqslant r_{3}$, то $r_{12}+r_{13}-r_{23} \geqslant r_{12}+r_{23}-r_{13} \geqslant r_{13}+r_{23}-r_{12}$, поэтому $r_{12} \geqslant r_{13} \geqslant r_{23}$. Окончательно имеем $\underline{R}^{+}=\max \left(r_{12}+r_{13}+r_{23}, 1+\right.$ $\left.\max \left(r_{12}, r_{13}, r_{23}\right)\right)$. Наконец,

$$
\begin{aligned}
\bar{R}^{+} & =r_{12}+r_{13}+r_{23}+1-\frac{r_{2}+r_{3}}{2}-\frac{r_{1}-r_{3}}{2} \\
& =r_{12}+r_{13}+r_{23}+1-r_{23}-\frac{\left(r_{12}+r_{13}-r_{23}\right)-\left(r_{13}+r_{23}-r_{12}\right)}{2} \\
& =r_{12}+r_{13}+r_{23}+1-r_{23}-\left(r_{12}-r_{23}\right) \\
& =1+\min \left(r_{13}+r_{23}, r_{12}+r_{23}, r_{12}+r_{13}\right) .
\end{aligned}
$$

Итак, мы доказали, что $\underline{R}^{+}=\max \left(r_{12}+r_{13}+r_{23}, 1+\max \left(r_{12}, r_{13}, r_{23}\right)\right)$ и $\bar{R}^{+}=1+\min \left(r_{13}+r_{23}, r_{12}+r_{23}, r_{12}+r_{13}\right)$, предполагая, что меры перенумерованы так, чтобы выполнялось соотношение $r_{1} \geqslant r_{2} \geqslant r_{3}$. Однако очевидно, что если вернуть мерам исходный порядок, то полученные формулы для $\underline{R}^{+}$и для $\bar{R}^{+}$не изменятся. Таким образом, доказано, что если $f(\vec{\mu}(\overrightarrow{\vec{C}}))=\sum_{i=1}^{3} \mu_{i}\left(C_{i}\right), \underline{R}^{+}=\inf _{\vec{\mu} \in D(\vec{r})} \sup _{\vec{C} \in \mathscr{C}(\Omega)} \sum_{i=1}^{3} \mu_{i}\left(C_{i}\right)$, $\bar{R}^{+}=\sup _{\vec{\mu} \in D(\vec{r})} \sup _{\vec{C} \in \mathscr{C}(\Omega)} \sum_{i=1}^{3} \mu_{i}\left(C_{i}\right)$, то выполняются следующие равенства:

$$
\begin{aligned}
& \underline{R}^{+}=\max \left(r_{12}+r_{13}+r_{23}, 1+\max \left(r_{12}, r_{13}, r_{23}\right)\right) \\
& \bar{R}^{+}=1+\min \left(r_{13}+r_{23}, r_{12}+r_{23}, r_{12}+r_{13}\right)
\end{aligned}
$$

Таким образом, теорема 1, сформулированная во введении, доказана.

П р и м е р 1. При $r_{i j}=r$ выражения для $\underline{R}^{+}$и $\bar{R}^{+}$принимают совсем простой вид: $\underline{R}^{+}=\max (1+r, 3 r), \bar{R}^{+}=1+2 r$.

3 а м е ч а н и е 2. Теорема 1 была доказана с помощью леммы 3 , которая опирается на лемму 1. Таким образом, доказательство теоремы 1 опирается на результаты, верные для произвольной функции $f$. Несложно показать, что условия теоремы 1 можно ослабить и требовать наличие лишь 6 непересекающихся множеств в $\sigma$-алгебре измеримого простраства $(\Omega, \Sigma)$. Для этого нужно доказывать теорему 1 не с помощью ссылки на общий случай, а «напрямую» (однако в этом случае доказательство уже не будет применимо к произвольной $f$ ). В самом деле, лемма 4 легко обобщается на случай произвольного $\Omega$, вследствие чего 
рассуждения, аналогичные лемме 2 , позволяют свести задачу о нахождении $\underline{R}^{+}$и $\bar{R}^{+}$к задаче о поиске экстремума по множеству $\Lambda_{6}$, которая решена выше.

3 а м е ч а н и е 3. Предъявим набор мер, на которых достигается экстремум в задаче о нахождении чисел $\underline{R}^{+}$и $\bar{R}^{+}$. Нам потребуется новое обозначение. Перенумеруем три числа $r_{12}, r_{13}, r_{23}$ в порядке неубывания: $r^{(1)} \leqslant r^{(2)} \leqslant r^{(3)},\left\{r^{(1)}, r^{(2)}, r^{(3)}\right\}=\left\{r_{12}, r_{13}, r_{23}\right\}$. Далее, рассмотрим тройку $\vec{\mu}^{*}$ мер на $\left(S_{3}, 2^{S_{3}}\right)$, заданную с помощью таблицы 1 . В силу непосредственной проверки можно убедиться, что тройка $\vec{\mu}^{*}$ является $\vec{r}$-согласованной (при этом $\vec{\mu}^{*} \in \Lambda_{6}$ ). Далее, если $\varepsilon_{1}=r^{(3)}-r^{(1)}$, то $\sup _{\vec{C} \in \mathscr{C}(\Omega)} \sum_{i=1}^{3} \mu_{i}^{*}\left(C_{i}\right)=\bar{R}^{+}$. Если же $\varepsilon_{1}=\min \left(r^{(2)}, 1-r^{(1)}\right)$, то $\sup _{\vec{C} \in \mathscr{C}(\Omega)} \sum_{i=1}^{3} \mu_{i}^{*}\left(C_{i}\right)=\underline{R}^{+}$. Отметим, что в силу леммы 4 в обоих случаях $\sup _{\vec{C} \in \mathscr{C}(\Omega)} \sum_{i=1}^{3} \mu_{i}^{*}\left(C_{i}\right)=\sum_{i=1}^{3} \mu_{i}^{*}\left(C_{i}^{*}\right)$ (множества $C_{i}^{*}, 1 \leqslant i \leqslant 3$, определены в таблице 1).

Таблица 1. Меры, на которых достигается экстремум.

\begin{tabular}{|c|c|c|c|c|}
\hline & $\sigma$ & $\mu_{1}^{*}$ & $\mu_{2}^{*}$ & $\mu_{3}^{*}$ \\
\hline \multirow{3}{*}{$C_{1}^{*}$} & $(123)$ & $1-r^{(1)}-\varepsilon_{1}$ & $1-r^{(1)}-\varepsilon_{1}$ & $1-r^{(1)}-\varepsilon_{1}$ \\
\cline { 2 - 5 } & $(132)$ & $r^{(3)}+r^{(2)}-r^{(1)}-\varepsilon_{1}$ & 0 & 0 \\
\hline \multirow{3}{*}{$C_{2}^{*}$} & $(213)$ & $r^{(1)}-r^{(3)}+\varepsilon_{1}$ & $r^{(1)}-r^{(3)}+\varepsilon_{1}$ & 0 \\
\cline { 2 - 5 } & $(231)$ & 0 & $r^{(3)}-\varepsilon_{1}$ & 0 \\
\hline \multirow{3}{*}{$C_{3}^{*}$} & $(312)$ & $r^{(1)}-r^{(2)}+\varepsilon_{1}$ & 0 & $r^{(1)}-r^{(2)}+\varepsilon_{1}$ \\
\cline { 2 - 5 } & $(321)$ & 0 & $\varepsilon_{1}$ & $r^{(2)}$ \\
\hline
\end{tabular}

Таким образом, мы предъявили набор мер, на которых достигается экстремум в задаче о нахождении чисел $\underline{R}^{+}$и $\bar{R}^{+}$.

6. Случай, когда количество мер больше трех. Несложно видеть, что результаты лемм 1 и 2 переносятся (с естественными изменениями) и на случай произвольного количества мер. А именно: пусть $M$ - множество вероятностных мер на измеримом пространстве $(\Omega, \Sigma)$. Для любых $n$ вероятностных мер $\mu_{1}, \mu_{2}, \ldots, \mu_{n}$ на $(\Omega, \Sigma)$ можно рассмотреть задачу различения $n \geqslant 3$ простых гипотез $H_{i}$ : наблюдение $\xi$ имеет распределение $\mu_{i}(i=1,2, \ldots, n)$. Каждому нерандомизированному критерию в этой задаче соответствуют измеримое разбиение $\vec{C}=\left\{C_{1}, C_{2}, \ldots, C_{n}\right\}$ пространства $\Omega\left(C_{i}: \bigsqcup_{i=1}^{n} C_{i}=\Omega\right.$, если наблюдение $\xi \in C_{i}$, то принимается гипотеза $\left.H_{i}, i=1,2, \ldots, n\right)$ и вероятности $\mu_{j}\left(C_{k}\right)$ принятия гипотезы $H_{k}$ в случае, когда верна гипотеза $H_{j}, j, k \in\{1,2, \ldots, n\}$. Совокупность всех разбиений $\Omega$ на $n$ непересекающихся измеримых подмножества обозначим $\mathscr{C}(\Omega)$. Далее, через $\vec{\mu}$ обозначим набор $\left(\mu_{1}, \mu_{2}, \ldots, \mu_{n}\right)$, через $\vec{\mu}(\vec{C})$ обозначим 
$\left(\mu_{1}\left(C_{1}\right), \mu_{1}\left(C_{2}\right), \ldots, \mu_{n}\left(C_{n}\right)\right) \in \mathbf{R}^{n^{2}}$. Будем считать, что качество критерия можно представить функцией $f(\vec{\mu}(\vec{C}))$ со значениями в $\mathbf{R}$; иногда будет использоваться обозначение $f\left(\left\{\mu_{j}\left(C_{k}\right)\right\}_{j, k=1}^{n}\right)$. Фиксируем такие числа $r_{i j}=r_{j i}, 1 \leqslant i, j \leqslant n, i \neq j$, что $0 \leqslant r_{i j} \leqslant 1,1 \leqslant i \neq j \leqslant n$, $r_{i j}+r_{j l} \geqslant r_{i l}, 1 \leqslant i, j, l \leqslant n, i \neq j \neq l \neq i$. Рассмотрим семейство всевозможных наборов из $n$ вероятностных мер на $\Sigma$, между которыми заданы попарные расстояния по вариации: $\rho\left(\mu_{i}, \mu_{j}\right)=r_{i j}, 1 \leqslant i<j \leqslant n$. Будем называть такие наборы мер $\vec{r}$-согласованными и обозначать это следующим образом: $\vec{\mu} \in D(\vec{r})$. Получим удобные выражения для чисел $\underline{R}=\inf _{\vec{\mu} \in D(\vec{r})} \sup _{\vec{C} \in \mathscr{C}(\Omega)} f(\vec{\mu}(\vec{C}))$ и $\bar{R}=\sup _{\vec{\mu} \in D(\vec{r})} \sup _{\vec{C} \in \mathscr{C}(\Omega)} f(\vec{\mu}(\vec{C}))$.

Для этого, как и ранее, построим множества $B_{i_{1}, i_{2}, \ldots, i_{n}}$ с помощью теоремы Хана. Заметим, что число множеств вида $B_{i_{1}, i_{2}, \ldots, i_{n}}$ равно $n$ ! Далее, введем множество $S_{n}$ перестановок индексов множеств $B_{i_{1}, i_{2}, \ldots, i_{n}}$ совершенно аналогично введению множества $S_{3}$. Множество $\Lambda_{n !}$ строится так же, как и множество $\Lambda_{6}$, и с помощью отображения $\phi_{n !}$ констру-

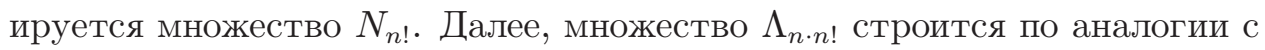
множеством $\Lambda_{18}$. С помощью отображения $\phi_{n \cdot n !}$ конструируется множество $N_{n \cdot n !}$. Тогда, повторяя рассуждения лемм 1 и 2 с естественными изменениями, получаем следующий результат.

Теорема 4. Пусть в б-алгебре измеримого пространства $(\Omega, \Sigma)$ содержится по крайней мере $n \cdot n$ ! непустых попарно не пересекаюшихся множеств. Тогда для любой функиии $f:[0,1]^{n^{2}} \rightarrow \mathbf{R}$ выполнень следуюшие равенства:

$\underline{R}=\inf _{\vec{x} \in N_{n !}} \max _{\vec{C} \in \mathscr{C}\left(S_{n}\right)} f\left(\left\{\sum_{\sigma \in C_{k}} x_{\sigma}^{(j)}\right\}_{j, k=1}^{n}\right), \quad \bar{R}=\sup _{\vec{x} \in N_{n \cdot n !}} f\left(\left\{\sum_{\sigma \in S_{n}} x_{\sigma}^{(j), k}\right\}_{j, k=1}^{n}\right)$.

Заметим, что для доказательства обобщения леммы 1 нам потребуется предположение о том, что в $\Sigma$ есть по меньшей мере $n \cdot n !$ непустых попарно не пересекающихся множеств, а для доказательства обобщения леммы 2 достаточно и наличия $n$ ! непустых попарно не пересекающихся множеств в $\Sigma$.

Далее, теорема 3 (аналогично теореме 2) может быть легко обобщена (с естественными изменениями) на случай произвольного количества мер.

Теперь покажем, что решение экстремальной задачи в классе мер без атомов может качественно отличаться от решения той же задачи в случае, когда наличие атомов у мер допускается (и $\Omega \neq \varnothing)$, притом даже в тех случаях, когда функция $f$ качества критерия непрерывна. Для этого рассмотрим величину

$$
\underline{L}:=\inf _{\vec{\mu} \in D(\vec{r})} \sup _{\vec{C} \in \mathscr{C}(\Omega)} h_{2}(\vec{\mu}(\vec{C}))=\inf _{\vec{\mu} \in D(\vec{r})} \sup _{\vec{C} \in \mathscr{C}(\Omega)} \min _{i \neq j}\left(\mu_{i}\left(C_{i}\right)-\mu_{i}\left(C_{j}\right)\right)
$$


(см. раздел «Обсуждение»). Заметим, что функция $h_{2}:[0,1]^{n^{2}} \rightarrow \mathbf{R}$ непрерывна. Далее, известен (см. [5, с. 67]) следующий факт: каким бы ни был набор из $n$ непрерывных мер, всегда найдется такое разбиение $\vec{C}^{*}$, для которого выполняется соотношение $\mu_{i}\left(C_{i}^{*}\right)-\mu_{i}\left(C_{j}^{*}\right) \geqslant 0$ при всех $1 \leqslant i, j \leqslant n, i \neq j$, т.е. $h_{2}\left(\vec{\mu}\left(\vec{C}^{*}\right)\right) \geqslant 0$. Отсюда следует, что для любого набора чисел $\left\{r_{i j}\right\}, 1 \leqslant i<j \leqslant n$, выполняется соотношение $\underline{L} \geqslant 0$ (в классе мер без атомов).

Теперь рассмотрим случай, когда $\Omega \neq \varnothing$ (т.е. когда в $\sigma$-алгебре измеримого пространства $(\Omega, \Sigma)$ содержится по крайней мере одно непустое множество) и $n \geqslant 2$. Так как $\Omega \neq \varnothing$, то существует $\omega_{0} \in \Omega$. Пусть $r_{i j}=0$ для всех $1 \leqslant i<j \leqslant N$. Рассмотрим набор из $n$ мер на $\Omega$, заданных следующим условием: $\mu_{i}(A)=1$ при $1 \leqslant i \leqslant n$ и при любых таких $A \in \Sigma$, что $\omega_{0} \in A$. Несложно видеть, что каким бы ни было разбиение $\vec{C}$, элемент $\omega_{0}$ попадет ровно в одно из множеств $C_{1}, C_{2}, \ldots, C_{n}$. Отсюда следует, что в данном случае $\underline{L}=-1$. Таким образом, существуют непрерывные функции $f$ качества критерия, для которых решение экстремальной задачи для $\underline{R}$ (в случае с $n$ мерами) в классе мер без атомов (более узком) качественно отличается от решения той же задачи в случае, когда наличие атомов у мер допускается (и $\Omega \neq \varnothing)$.

7. Результаты для случая с рандомизацией. Пусть $\mathbb{E}=$ $\left(\Omega, \mathbb{F},\left(P_{\theta}\right)_{\theta \in \Theta}\right)$ - статистическая модель, причем $\Theta=\{1,2,3\}$ и пространство $D$ решений совпадает с множеством $\Theta$, функция потерь $L(\theta, d)=\mathbb{I}_{\theta \neq d}$, функция $\phi=\left(\phi_{1}, \phi_{2}, \phi_{3}\right)$ задает рандомизированный критерий $\left(\phi_{i}: \Omega \rightarrow[0,1]\right.$ измеримы для каждого $1 \leqslant i \leqslant 3$ и $\left.\sum_{i=1}^{3} \phi_{i}=1\right)$ следующим образом: $\phi_{i}(\omega)$ - это вероятность принять решение под номером $i$, если происходит $\omega$. Множество таких критериев обозначим $\Phi$, а множество нерандомизированных критериев обозначим $\Phi^{*}$. В «рандомизированном» случае байесовский риск $p$, соответствующий равномерному распределению на $\Theta$, равен $\inf _{\phi \in \Phi} \frac{1}{3} \sum_{i=1}^{3} \mathbf{E}_{P_{i}}\left(1-\phi_{i}\right)$. Удобнее рассматривать величину $\bar{a}:=1-p=\sup _{\phi \in \Phi} \frac{1}{3} \sum_{i=1}^{3} \mathbf{E}_{P_{i}} \phi_{i}$. Далее, пусть $\bar{a}^{*}=\sup _{\phi \in \Phi^{*}} \frac{1}{3} \sum_{i=1}^{3} \mathbf{E}_{P_{i}} \phi_{i}$. Ниже мы покажем, что $\bar{a}=\bar{a}^{*}$, откуда следует, что неулучшаемой оценкой величины $\bar{a}$ является $\bar{R}^{+}$(в силу того, что неулучшаемой оценкой для величины $\bar{a}^{*}$ является $\bar{R}^{+}$). Верна следующая теорема.

Теорема 5. Выполнень следующие равенства:

$$
\begin{aligned}
& \sup _{\vec{\mu} \in D(\vec{r})} \sup _{\phi \in \Phi} \sum_{i=1}^{3} \mathbf{E}_{P_{i}} \phi_{i}=\sup _{\vec{\mu} \in D(\vec{r})} \sup _{\phi \in \Phi^{*}} \sum_{i=1}^{3} \mathbf{E}_{P_{i}} \phi_{i}=\bar{R}^{+}, \\
& \inf _{\vec{\mu} \in D(\vec{r})} \sup _{\phi \in \Phi} \sum_{i=1}^{3} \mathbf{E}_{P_{i}} \phi_{i}=\inf _{\vec{\mu} \in D(\vec{r})} \sup _{\phi \in \Phi^{*}} \sum_{i=1}^{3} \mathbf{E}_{P_{i}} \phi_{i}=\underline{R}^{+} .
\end{aligned}
$$


Д о к а з а т е л ь с т в о. Фиксируем тройку мер $\vec{\mu} \in D(\vec{r})$ и $\phi=$ $\left(\phi_{1}, \phi_{2}, \phi_{3}\right) \in \Phi$. Пусть $\mu:=\frac{1}{3} \sum_{i=1}^{3} \mu_{i}$ и $\mathfrak{z}_{i}:=d \mu_{i} / d \mu$. Тогда

$$
\sum_{i=1}^{3} \int_{\mathbf{R}} \phi_{i} d \mu_{i}=\int_{\mathbf{R}} \sum_{i=1}^{3} \phi_{i} \mathfrak{z}_{i} d \mu \leqslant \int_{\mathbf{R}} \sum_{i=1}^{3} \phi_{i}\left(\max _{j} \mathfrak{z}_{j}\right) d \mu=\int_{\mathbf{R}} 1\left(\max _{j} \mathfrak{z}_{j}\right) d \mu .
$$

Если $\phi_{i} \mathfrak{z}_{i}=\phi_{i}\left(\max _{j} \mathfrak{z}_{j}\right)$, т.е. $\phi_{i}\left(\mathfrak{z}_{i}-\max _{j} \mathfrak{z}_{j}\right)=0$, то в предыдущей формуле достигается равенство. Пусть

$$
\begin{gathered}
C_{1}:=\left\{\max _{j} \mathfrak{z}_{j}=\mathfrak{z}_{1}\right\}, \quad C_{2}:=\left\{\max _{j} \mathfrak{z}_{j}=\mathfrak{z}_{2}\right\} \bigcap\left(\Omega \backslash C_{1}\right), \\
C_{3}:=\left(\Omega \backslash C_{1}\right) \backslash C_{2}, \quad \widehat{\phi}_{i}(\omega):=I_{C_{i}}(\omega) .
\end{gathered}
$$

Несложно показать, что $\widehat{\phi}_{i}\left(\mathfrak{z}_{i}-\max _{j} \mathfrak{z}_{j}\right)=0$. Таким образом, при $\phi_{i}=\widehat{\phi}_{i}$ неравенство (20) переходит в равенство (причем функции $\widehat{\phi}_{i}$ принимают только значения 0 и 1$)$. Следовательно, $\widehat{\phi}:=\left(\widehat{\phi}_{1}, \widehat{\phi}_{2}, \widehat{\phi}_{3}\right) \in \Phi^{*} \subset \Phi$ и $\sup _{\phi \in \Phi} \sum_{i=1}^{3} \int_{\mathbf{R}} \phi_{i} d \mu_{i}=\sum_{i=1}^{3} \int_{\mathbf{R}} \widehat{\phi}_{i} d \mu_{i}$. Поэтому $\sup _{\phi \in \Phi} \frac{1}{3} \sum_{i=1}^{3} \mathbf{E}_{P_{i}} \phi_{i}=$ $\sup _{\phi \in \Phi^{*}} \frac{1}{3} \sum_{i=1}^{3} \mathbf{E}_{P_{i}} \phi_{i}$ (т.е. $\left.\bar{a}=\bar{a}^{*}\right)$. Значит,

$$
\begin{aligned}
& \sup _{\vec{\mu} \in D(\vec{r}))} \sup _{\phi \in \Phi} \sum_{i=1}^{3} \mathbf{E}_{P_{i}} \phi_{i}=\sup _{\vec{\mu} \in D(\vec{r})} \sup _{\phi \in \Phi^{*}} \sum_{i=1}^{3} \mathbf{E}_{P_{i}} \phi_{i}, \\
& \inf _{\vec{\mu} \in D(\vec{r})} \sup _{\phi \in \Phi} \sum_{i=1}^{3} \mathbf{E}_{P_{i}} \phi_{i}=\inf _{\vec{\mu} \in D(\vec{r})} \sup _{\phi \in \Phi^{*}} \sum_{i=1}^{3} \mathbf{E}_{P_{i}} \phi_{i} .
\end{aligned}
$$

Осталось заметить, что

$$
\begin{aligned}
& \sup _{\vec{\mu} \in D(\vec{r})} \sup _{\phi \in \Phi^{*}} \sum_{i=1}^{3} \mathbf{E}_{P_{i}} \phi_{i}=\sup _{\vec{\mu} \in D(\vec{r})} \sup _{\vec{C} \in \mathscr{C}(\Omega)} \sum_{i=1}^{3} \mu_{i}\left(C_{i}\right)=\bar{R}^{+}, \\
& \inf _{\vec{\mu} \in D(\vec{r})} \sup _{\phi \in \Phi^{*}} \sum_{i=1}^{3} \mathbf{E}_{P_{i}} \phi_{i}=\inf _{\vec{\mu} \in D(\vec{r})} \sup _{\vec{C} \in \mathscr{C}(\Omega)} \sum_{i=1}^{3} \mu_{i}\left(C_{i}\right)=\underline{R}^{+}
\end{aligned}
$$

по определению множества $\Phi^{*}$. Значит,

$$
\begin{aligned}
& \sup _{\vec{\mu} \in D(\vec{r})} \sup _{\phi \in \Phi} \sum_{i=1}^{3} \mathbf{E}_{P_{i}} \phi_{i}=\sup _{\vec{\mu} \in D(\vec{r})} \sup _{\phi \in \Phi^{*}} \sum_{i=1}^{3} \mathbf{E}_{P_{i}} \phi_{i}=\bar{R}^{+}, \\
& \inf _{\vec{\mu} \in D(\vec{r})} \sup _{\phi \in \Phi} \sum_{i=1}^{3} \mathbf{E}_{P_{i}} \phi_{i}=\inf _{\vec{\mu} \in D(\vec{r})} \sup _{\phi \in \Phi^{*}} \sum_{i=1}^{3} \mathbf{E}_{P_{i}} \phi_{i}=\underline{R}^{+},
\end{aligned}
$$

что и требовалось. Теорема 5 доказана.

3 а м е ч а н и е 4 . Теорема 5 очевидным образом обобщается на случай произвольного конечного числа мер.

3 а м е ч а н и е 5 . Теорема 5 доказывает, что в случае, когда под функцией качества критерия понимается сумма вероятностей ошибок, рандомизация не позволяет улучшить оптимальный нерандомизированный критерий. 


\section{СПИСОК ЛИТЕРАТУРЫ}

1. Ширяев А.Н. Вероятность, т. 1. М.: МНЦМО, 2004, 575 с.

2. Гущин A.A. О лемме Фано и аналогичных неравенствах для минимаксного риска. - Теорія имовір. та матем. статист., 2002, № 67, с. 26-37.

3. Birge L. A new look at an old result: Fano's lemma. - Prepublication $n^{0} 632 \mathrm{du}$ Labotatoire de Probabilites \& Modeles Aleatores, Univesites de Paris 6 \& 7, January 2001.

4. Elton J., Hill T.P., Kertz R. Optimal-partitioning inequalities for nonatomic probability measures. - Trans. Amer. Math. Soc., 1986, v. 296, № 2, p. 703-725.

5. Robertson J., Webb W. Cake-cutting Algorithms. Be Fair, if You Can. Natick: Massachusetts, 1998.

6. Fano R. M. Class notes for transmission of information, Course 6.574. Cambridge, MA: MIT, 1952.

7. Thompson $W$. Fair allocation rules. - University of Rochester, working paper No. 539, December 2007.

8. Hill T.P. Partitioning inequalities in probability and statistics. - Stochastic Inequalities, 1993, v. 22, p. 116-132.

9. Ben-Bassat M. f-Entropies, probability of error, and feature selection. - Inform. Contr., 1978, v. 39, p. 227-242.

10. Golic J. On the relationship between the information measures and the Bayes probability of error. - J. IEEE Transac. Inform. Theory, 1987, v. 33, № 5, p. 681693.

11. Golic J. On the Relationship Between the Separability Measures and the Bayes Probability of Error. - J. IEEE Transac. Inform. Theory, 1987, v. 33, № 5, p. 694-701.

Поступила в редакцию 27.X.2015 